\title{
Astrocytes regulate inhibition in Fragile X Syndrome
}

Maham Rais $^{1}$, Anna O. Kulinich ${ }^{1}$, Victoria Wagner ${ }^{1,2}$, Walker Woodard ${ }^{1}$, Xinghao S. Shuai ${ }^{1}$,

Samantha N. Sutley ${ }^{1}$, Jamiela Kokash ${ }^{2}$, Maija Castren ${ }^{4}$, Khaleel A. Razak ${ }^{2,3}$, and Iryna M.

Ethell ${ }^{1,2 *}$

${ }^{1}$ Division of Biomedical Sciences and Biomedical Sciences Graduate Program, School of

Medicine, University of California Riverside, Riverside, CA 92521

${ }^{2}$ Neuroscience Graduate Program, University of California Riverside, Riverside, CA 92521

${ }^{3}$ Department of Psychology, University of California Riverside, Riverside, CA 92521

${ }^{4}$ Department of Physiology, University of Helsinki, Helsinki, Finland

*Corresponding Author:

Iryna M. Ethell, $\mathrm{PhD}$

Division of Biomedical Sciences

Email: iryna.ethell@medsch.ucr.edu

Phone: 951-827-2186

Number of Figures: 8

Supplementary Figures: 8

Supplementary Tables: 11

We report no conflict of interest. 


\begin{abstract}
Fragile X syndrome (FXS) is a leading genetic cause of autism-like symptoms that include sensory hypersensitivity and cortical hyperexcitability. Recent observations in humans and Fmrl knockout (KO) animal models of FXS suggest abnormal GABAergic signaling. As most studies focused on neuron-centered mechanisms, astrocytes' contribution to abnormal inhibition is largely unknown. Here we propose a non-neuronal mechanism of abnormal inhibitory circuit development in FXS. Astrocyte-specific deletion of Fmrl during postnatal period leads to increased astrocytic GABA levels, but negatively impacts synaptic GABA receptors and parvalbumin (PV) cell development. Developmental deletion of Fmr 1 from astrocytes also affects communications between excitatory neurons and PV cells, impairing sound-evoked gamma synchronization in the cortex, while enhancing baseline and on-going sound-evoked EEG power, and leading to increased locomotor activity and altered social behaviors in adult mice. These results demonstrate a profound role of astrocytic FMRP in the development of inhibitory circuits and shaping normal inhibitory responses.
\end{abstract}

Keywords: Autism, Fragile X Syndrome, Neurodevelopmental Disorders, Astrocytes, GABAergic Signaling, Parvalbumin Inhibitory Interneurons, Electrocortical Activity, Cortical Circuits 


\section{Introduction}

Fragile X Syndrome (FXS) is the most common genetic form of autism spectrum disorders (ASD) ${ }^{1}$. FXS is usually caused by a CGG repeat expansion in 5'-untranslated region of the Fragile $\mathrm{X}$ mental retardation $1(F m r l)$ gene followed by gene methylation, loss of Fragile $\mathrm{X}$ Mental Retardation Protein (FMRP), translational dysregulation, and abnormal protein synthesis $^{2,3}$. Prominent symptoms of FXS include intellectual disability, repetitive behaviors, social communication deficits, and sensory hypersensitivity ${ }^{4,5}$, particularly in the auditory domain $^{6-8}$. Sensory hypersensitivity is observed in both humans with FXS and the mouse models of FXS and can stem from hyperexcitable cortical circuits ${ }^{5-8}$.

The Fmrl knockout (KO) mouse is an established FXS model that is well suited to study cellular mechanisms of cortical hyperexcitability. FMRP loss increases network-level excitability in the rodent cortex through impaired inhibition and altered excitatory/inhibitory (E/I) balance ${ }^{9}$ that most likely affects neural synchrony ${ }^{10,11}$. The Fmrl KO mice shows decreased density and function of parvalbumin (PV)-expressing inhibitory interneurons ${ }^{12-15}$, reduced gammaaminobutyric acid (GABA) receptor levels, altered GABA metabolism, and overall decreased GABAergic input in several areas of the brain ${ }^{16-19}$. Studies to date suggest that FMRP loss affects the communications between excitatory pyramidal neurons and inhibitory interneurons ${ }^{12,20}$ resulting in abnormal E/I balance ${ }^{21}$. Although FMRP is involved in the regulation of synaptic function and neural communication ${ }^{22-24}$, little is known how different cell types may contribute to the deficits.

Astrocytes are known to control neural communication not only by regulating glutamate metabolism, but also through uptake and synthesis of GABA, thereby influencing GABAergic 
transmission $^{25,26}$. However, most studies of astrocytes are focused on excitatory synapses and pyramidal cells (PC), leaving inhibitory synapses, and GABAergic interneurons less explored ${ }^{27}$. Fmrl KO astrocytes are known to trigger FXS-like changes in WT excitatory neurons ${ }^{28-30}$. The enhanced excitability was also linked to reduced levels of glutamate transporter in FMRPdeficient astrocytes and reduced glutamate uptake by the astrocytes ${ }^{28,31}$. Whether astrocytes contribute to abnormal inhibitory cell development and circuit hyperexcitability by altering GABAergic signaling has not been investigated in FXS.

Therefore, the main goal of this study was to determine whether cell-specific deletion of Fmrl from astrocytes during the critical developmental periods of excitatory and inhibitory circuit refinement ${ }^{32-35}$ would alter GABAergic signaling and PV cell development leading to cortical hyperexcitability and behavioral alterations. In the present study, we used a multidisciplinary approach including transcriptional, cellular, molecular, electrophysiological, and behavioral methods to delineate the astrocyte-mediated mechanisms of abnormal inhibition in FXS. We also utilized the analysis of translationally relevant electroencephalogram (EEG) phenotypes that are remarkably similar between mouse model of FXS and human condition across different brain areas implicated in FXS-associated behaviors ${ }^{36}$. Interestingly, specific deletion of Fmrl from astrocytes resulted in more severe deficits than the effects of Fmrl deletion from $\mathrm{PC}^{14,37}$. Our findings provide novel insight into the role of astrocytic FMRP in the development of cortical circuits and suggest non-neuronal mechanism of abnormal inhibitory circuit development in FXS with potential for translating the results of the mouse study into successful clinical trials.

\section{Results}


In the current study, we investigated the contribution of astrocytes to abnormal inhibition in FXS. We examined whether FMRP deletion from astrocytes during the developmental period of inhibitory circuit refinement affects (1) PV cell development and connectivity; (2) maturation of baseline and sound-evoked responses in the adult auditory cortex (AuC) and frontal cortex (FC); and (3) mouse behaviors, such as locomotor activity, anxiety and sociability.

\section{Astrocyte-specific FMRP deletion during postnatal developmental period}

To achieve specific Fmrl deletion in astrocytes, ERT2-Cre ${ }^{G F A P} F m r 1^{\text {flox } / \mathrm{y}}$ conditional KO (cKO) (Group 1) were generated and ERT2-Cre ${ }^{G F A P}$ wild-type (WT) mice were used as controls (Ctrl WT; Fig. 1a). For analysis of FMRP levels in astrocytes, tdTomato was expressed in astrocytes using Rosa-CAG-LSL-tdTomato reporter mice to generate tdTomatoERT2$\mathrm{Cre}^{G F A P} F m r 1^{\text {flox/y }} \mathrm{cKO}$ mice and tdTomatoERT2-Cre ${ }^{G F A P} \mathrm{Ctrl}$ WT mice (Group 2) (Fig. 1a). For analysis of connectivity of parvalbumin (PV) inhibitory interneurons, tdTomato was expressed under Pvalb promoter in Pvalb-tdTomato-ERT2-Cre ${ }^{G F A P} F m r I^{\text {flox/y }} \mathrm{cKO}$ and $P$ valb-tdTomatoERT2-Cre ${ }^{G F A P}$ Ctrl WT mice (Group 3) (Fig. 1a). In all groups, Ctrl WT and cKO mice received tamoxifen at postnatal day (P)14 intraperitoneally (IP, $0.5 \mathrm{mg}$ in $5 \mathrm{mg} / \mathrm{ml}$ of 1:9 ethanol/sunflower seed oil solution) once a day for 5 days, and analysis was performed at P28 or P60 (adult) (Fig. 1a).

We observed an overall reduction in Fmrl mRNA levels in the hippocampus and cortex of P28 cKO mice using qRT-PCR (Fig 1b, Supplementary Fig. 1, Supplementary Table 1). To confirm specific ablation of Fmrl in astrocytes, FMRP immunoreactivity was analyzed in the

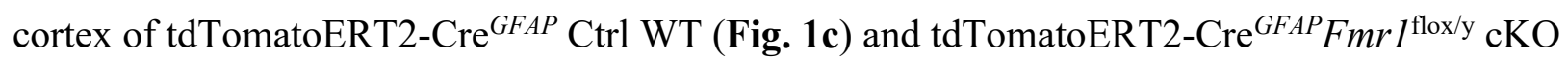
mice at P28 (Fig. 1d). Although no significant genotype differences were observed in overall 
FMRP-positive cell density (Fig. 1e) or FMRP levels in all FMRP-positive cells (Fig. 1f), FMRP immunoreactivity was significantly decreased in cortical astrocytes of $\mathrm{cKO}$ mice compared to Ctrl WT (Fig. 1g, Supplementary Table 1). These results confirm successful deletion of FMRP specifically from developing astrocytes during the postnatal P14-P28 window, without affecting FMRP levels in neurons.

\section{Deletion of Fmr1 from astrocytes leads to impaired development of PV-expressing inhibitory cells.}

We first examined whether FMRP deletion from astrocytes during postnatal period of inhibitory circuit refinement affects PV cell development and connectivity. PV-expressing cells are fast-spiking inhibitory interneurons that provide temporal precision to excitatory activity and their loss or hypofunction may contribute to cortical hyperexcitability in individuals with autism and mouse models of autism, including FXS, 13, 15, 38-43. Interestingly, we observed a significant decrease in PV mRNA levels in the developing hippocampus and cortex following FMRP deletion from astrocytes (Fig. 2a). While overall PV protein levels were also significantly reduced in the cortex, we did not observe significant differences in the hippocampus, potentially due to greater fluctuations of PV levels in the hippocampus (Fig. 2b, Supplementary Table 2).

The downregulation in cortical PV levels may suggests an overall reduced recruitment of PV cells by excitatory neurons, which may in turn affect perisomatic inhibitory innervation of PC. Therefore, we next detected inhibitory perisomatic synaptic sites with immunostaining against VGAT and gephyrin, and analyzed their association with PV-positive pre-synaptic boutons visualized with tdTomato in Pvalb-tdTomato-ERT2-Cre ${ }^{G F A P} F m r 1^{\text {flox/y }} \mathrm{cKO}$ and PvalbtdTomato-ERT2-Cre ${ }^{G F A P}$ Ctrl WT male mice (Fig. 2c). We observed a significant decrease in 
vGAT/Gephyrin, PV/Gephyrin, and percent of PV-positive inhibitory synaptic sites in the superficial layers (L2-4) of AuC in cKO mice compared to Ctrl WT, suggesting reduced PV neuron innervation of PC (Fig. 2d-f, Supplementary Table 3). Next, we examined the excitatory innervation of PV cells by PC. To determine the number of excitatory synapses on tdTomato-expressing PV inhibitory neurons, we analyzed co-localization of presynaptic vGlut1 and postsynaptic PSD95 puncta in the auditory cortex of Pvalb-tdTomato-ERT2-

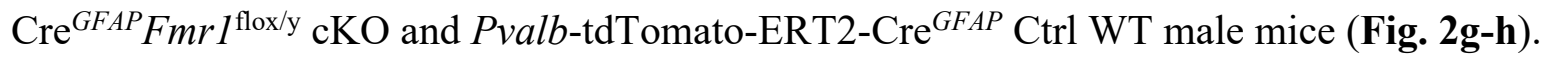
There is a significant decrease in vGlut1/PSD95 puncta on PV cells in L2-4 AuC of cKO mice compared to Ctrl WT (Fig. 2h-i, Supplementary Table 3). The impaired connectivity between excitatory and PV neurons during the P14-P28 critical period of inhibitory circuit refinements is most likely responsible for an overall decrease in the numbers of PV-expressing cells observed in L2-4 AuC of adult cKO mice (Fig. 2j-k, Supplementary Table 4). In addition, WFA+ PNN cell density and PV/PNN co-localization were significantly reduced in L4 AuC of cKO mice (Fig. $\mathbf{2 j - k}$ ), similar to what was previously seen in adult excitatory neuron-specific Fmrl KO mice ${ }^{14}$. Taken together, our results suggest that the loss of astrocytic Fmrl results in reduced excitatory synapse formation onto PV neurons and lower number of PV-expressing cells (Fig. 2i). The reduced excitatory drive onto PV inhibitory neurons in the developing cortex may be responsible for reduced expression of PV and impaired perisomatic inhibitory innervation of PC, resulting in an overall increased cortical excitability in astrocyte-specific Fmrl cKO mice.

\section{Enhanced baseline EEG gamma band power and impaired cross-frequency power coupling in the auditory and frontal cortex of adult cKO mice.}


The impaired PV-PC cell connectivity is predicted to alter neural oscillations ${ }^{44}$.

Therefore, we next tested EEG responses in adult astrocyte-specific cKO mice. Power spectral density of baseline (no sound simulation) EEG responses was calculated in AuC and FC of freely-moving Ctrl WT (n=10) and cKO (n=9) mice (Supplementary Fig. 1e). Enhanced high frequency gamma oscillations are apparent from the examples of 2 s raw EEG traces (Fig. 3a) and group average power spectral densities from 5-min EEG recordings in both AuC (Fig. 3b, c) and FC (Fig. 3b, e) of cKO mice compared to Ctrl WT. Statistical analysis was performed using a one-way MANCOVA approach with percentage time the mouse spent moving as a covariate to compare genotype mean differences on 6 bands per region: Delta (1-4 Hz), Theta (4-8H z), Alpha (8-13 Hz), Beta (13-30 Hz), Low Gamma (30-55 Hz) and High Gamma (65-100 Hz). We confirmed assumptions of equality of covariance using Box's M, p $=0.071$ (for Ctrl WT vs. cKO $\mathrm{AuC}$ ) and $\mathrm{p}=0.319$ (for Ctrl WT vs cKO FC). Levene's test of equality of error variance showed no difference in variance between genotypes in any of the bands (all $p>0.05)$. A significant effect of genotype (AuC: Ctrl WT vs. cKO: Pillai's Trace $=0.748, \mathrm{p}=0.020 ; \mathbf{F C}$ : Ctrl WT vs. cKO: Pillai's Trace $=0.707, \mathrm{p}=0.038$ ) was observed across all 6 of the combined frequency variables, which included mouse movement as a covariate. Further post-hoc comparisons identified significant differences between genotypes in gamma power, including low and high gamma power, in both AuC (Fig. 3d) and FC (Fig. 3f) after correction for multiple comparisons

\section{(Supplementary Table 5).}

Human EEG studies also report power abnormalities in delta, theta and alpha frequencies, as well as impaired coupling between low- and high-frequency oscillations in individuals with FXS and $\mathrm{ASD}^{45,46}$. To assess the relationship between power across different frequencies and/or regions, a Pearson's correlation analysis was done using the same approach that was used in a 
clinical study of $\mathrm{FXS}^{46}$ (Fig. 3g). While there was a positive correlation in power coupling of the AuC Delta/AuC Gamma (D1/G1), AuC Theta/AuC Gamma (T1/G1), and AuC Alpha/AuC Gamma (A1/G1) in Ctrl WT mice, the correlation was lost in astrocyte-specific Fmrl cKO mice (Fig. 3g, Supplementary Table 6). Results also show a positive correlation in power coupling of the Delta/Gamma in the FC of Ctrl WT mice, but not in cKO mice (D2/G2, Fig. 3g, Supplementary Table 6). Similar results were observed for delta and theta coupling to gamma between AuC and FC (D1/G2, T1/G2 for AuC to FC and D2/G1 for FC to AuC correlations, Fig. 3g, Supplementary Table 6). Overall, these findings indicate that abnormalities in baseline gamma power and EEG power coupling between low- and high-frequency oscillations in the AuC and FC, suggesting that impaired connectivity between PC and PV cells most likely influence neuronal activity, leading to abnormal synchronization of neural activity in the cortex.

\section{Postnatal deletion of Fmr1 from astrocytes reduces fidelity of temporal processing in the auditory and frontal cortex of adult mice.}

Given that PV neuron activity is vital for timing fidelity of responses, we tested the hypothesis that the ability of the cortex to mount a consistent phase locked response to repeated time-varying stimuli would be impaired in astrocyte-specific cKO mice. Using a 'chirp' stimulus, previous studies in rodents and humans have shown a reduction in inter-trial phase coherence (ITPC) in FXS. The chirp is a 2-s broadband noise stimulus whose amplitude is modulated (100\% depth) by a sinusoid of linearly increasing frequencies from 1-100 Hz. The ability of the neural generators to consistently phase lock to this time-varying signal is a measure of fidelity in temporal processing. After repeated chirp presentations (300 trials for up, 300 for down), the ITPC was calculated across trials in the time $\mathrm{X}$ frequency domain using Morlet 
Wavelet analysis as previously described ${ }^{14,37}$. Here, the results are presented only for 'up' chirp because the chirp responses were not affected by the direction of frequency change. After grand average ITPC was calculated for each group, means for Ctrl WT mice $(n=10)$ were subtracted from the means for cKO mice $(\mathrm{n}=9)$ (AC, Fig. 4a; FC, Fig. 4b). For statistical comparisons, non-parametric cluster analysis was used to determine contiguous regions in the time $\mathrm{X}$ frequency domain that were statistically different between genotypes. We observed a significant decrease in ITPC at beta (13-30 Hz) and gamma frequencies (30-100 Hz) in AuC (Fig. 4a) and FC (Fig. 4b) of cKO mice. These data indicate that postnatal deletion of FMRP from astrocytes results in the development of gamma synchronization deficits in adult mice as was previously observed in global Fmrl KO mice ${ }^{37}$.

Next, we investigated non-phase locked single trial power (STP) during the chirp stimulation period (Fig. 4c, d; Supplementary Fig. 2), because any increase in gamma power during the duration of acoustic stimulation may decrease temporally consistent responses to the dynamic chirp stimulus. cKO mice showed a significant increase in non-phase locked background gamma power in AuC (Fig. 4c) and FC (Fig. 4d) in the entire gamma band ( 30$100 \mathrm{~Hz}$ ), similar to the findings in the global Fmrl KO mouse ${ }^{37}$ and in humans with $\mathrm{FXS}^{47}$. These results suggest that astrocytes might indirectly regulate background broadband gamma power during epochs of sounds stimulation by influencing the activity of fast-spiking inhibitory interneurons.

\section{Postnatal deletion of Fmrl from astrocytes amplifies on-going response to broadband noise in the cortex of adult cKO mice.}


To compare sound-evoked responses to trains of brief (100 ms) broadband noise stimuli (10 stimuli per train, 65-70 dB SPL, 100 repetitions of each train), we tested both a nonhabituating repetition rate $(0.25 \mathrm{~Hz})$ and a habituating rate of sound presentation $(4 \mathrm{~Hz})^{14,48}$. Example traces are shown for the first stimulus in the $0.25 \mathrm{~Hz}$ train (Fig. 5a) and the first 4 stimuli in the $4 \mathrm{~Hz}$ train (Fig. 5b) in AuC and FC of Ctrl WT and cKO. We measured the power of the onset and on-going responses for each repetition rate (Supplementary Fig. 3) and observed enhanced power of the response in gamma range in the $\mathrm{AuC}$, but not $\mathrm{FC}$, during the onset of single sound presentation at $0.25 \mathrm{~Hz}$ repletion rate and all four sounds in $4 \mathrm{~Hz}$ sound train (Fig. 5c, d; Supplementary Fig. 3). In addition, both the AuC and FC exhibited enhanced on-going power across frequencies after sound presentation at $0.25 \mathrm{~Hz}$ rate, indicating increased continuous cortical activity potentially due to impaired inhibition ${ }^{49}$. Lastly, in both the AuC and FC, increased onset and on-going power is observed at $\sim 10-30 \mathrm{~Hz}$ frequencies during the presentation of all four sounds in $4 \mathrm{~Hz}$ sound train (Fig. 5d). This increase of continuous oscillation power is visually apparent in the example traces shown in Fig. $\mathbf{5 b}$, where cKO shows higher amplitude oscillations throughout the sound train compared to Ctrl WT group. These results demonstrate high sound-induced power during both sound onset and on-going responses in the alpha, beta and gamma frequency range in adult mice following postnatal FMRP deletion from astrocytes with some differences between $\mathrm{AuC}$ and FC. These abnormal responses indicate cortical hypersensitivity to sounds mediated by loss of FMRP in astrocytes.

\section{Postnatal deletion of Fmr1 from astrocytes alters expression of genes involved in}

\section{GABAergic transmission.}


To determine if postnatal deletion of Fmrl from astrocytes affects expression of glial and neuronal genes associated with GABAergic transmission, we analyzed mRNA levels of selected genes in the hippocampus and cortex of P28 Ctrl WT and cKO mice (Supplementary Fig. 1). Hippocampal mRNA expression was first analyzed with NanoString “Glial Profiling” panel (Supplementary Fig. 4a). Analysis identified 81 differentially expressed genes (DEGs), including 66 downregulated and 15 upregulated DEGs (Supplementary Fig. 4-6). Although some of the 81 DEGs were glial-specific, most of DEGs were associated with synaptic signaling, regulation of synaptic plasticity, GABAergic synaptic transmission and GABA receptor complex (Supplementary Fig. 5a-c). Further Gene Ontology (GO) analysis indicated strong association of DEGs with the "Synaptic vesicle cycle" and "GABAergic synapse" pathways in the Kyoto Encyclopedia of Genes and Genomes (KEGG) pathway database (Supplementary Fig. 5d). As previous studies have indicated that $\mathrm{GABA}_{\mathrm{A}}$ receptors $\left(\mathrm{GABA}_{\mathrm{A}} \mathrm{R}\right)$ levels are affected in $\mathrm{FXS}^{17}$, 19,50-52, we further analyzed the specific genes encoding subunits of GABA $\mathrm{R}$ in the hippocampus followed by qRT-PCR analysis of mRNA expression in the cortex.

NanoString normalized gene expression data indicate that some, but not all, genes associated with GABAergic transmission were dysregulated (Fig. 6a). We found no significant differences in mRNA levels of GABA synthesizing enzymes Gad1 and Gad2 in the hippocampus between genotypes (Fig. 6b). However, mRNA expression of Gabra1, Gabra3 and Gabrg2 genes encoding synaptic $\mathrm{GABA}_{\mathrm{A}} \mathrm{R}$ subunits was significantly decreased in the hippocampus (NanoString) and cortex (qRT-PCR) of cKO mice compared to Ctrl WT (Fig. 6c,

Supplementary Table 7). Interestingly, we found no changes in the expression of Gabra4, Gabra5 and Gabrd genes that encode primarily extrasynaptic $\mathrm{GABA}_{\mathrm{A}} \mathrm{R}$ subunits (Fig. 6d), as well as Gabrb3 that is associated with both synaptic and extrasynaptic GABA $\mathrm{R}$ (not shown). 
Consistent with the reduced mRNA levels, we also observed a significant decrease in the protein levels of synaptic $\mathrm{GABA}_{\mathrm{A}} \mathrm{R} \gamma 2$ subunit but not $\mathrm{GABA}_{\mathrm{A}} \mathrm{R} \alpha 5$ subunit in $\mathrm{cKO}$ mice compared to Ctrl WT (Fig. 6e-f, Supplementary Table 7). Taken together, these data indicate that postnatal deletion of Fmrl from astrocytes affects the expression of synaptic $\mathrm{GABA}_{\mathrm{A}} \mathrm{R}$ subunits, while the levels of extrasynaptic $\mathrm{GABA}_{\mathrm{A}} \mathrm{R}$ subunits remain unchanged.

\section{Astrocytic GABA is significantly increased in the hippocampus and cortex of cKO mice.}

As FMRP is deleted only from astrocytes the changes in the levels of synaptic $\mathrm{GABA}_{\mathrm{A}} \mathrm{R}$ gene expression may be a compensatory mechanism to offset the increase in extracellular GABA levels. Our data suggest an astrocyte-mediated mechanism of GABA dysregulation and show higher GABA immunoreactivity in glutamate synthetase (GS)-positive Fmrl-deficient astrocytes in the hippocampus and cortex of cKO mice (Fig. 7a-b, Supplementary Table 8). The increase is likely a result of enhanced GABA synthesis in Fmrl KO astrocytes as we also observe higher levels of GABA and GABA-synthesizing enzyme GAD65/67, but not Aldha1, in cultured astrocytes isolated from Fmrl KO mice compared to WT astrocytes (Fig. 7c-d). While we observed no changes in total mRNA levels of gad1/2 in the hippocampus and cortex of astrocyte Fmrl cKO mice (Fig. 6b), protein levels of GAD65/67 were significantly upregulated in Fmrl KO astrocytes compared to WT astrocytes (Fig. 7c). We also observed higher GABA immunoreactivity in glutamate synthetase (GS)-positive Fmrl deficient astrocytes compared to WT astrocytes in the astrocyte-enriched cultures (Fig. 7d), suggesting that the increase is a result of enhanced GABA synthesis in astrocytes instead of neuronal GABA uptake. Consistent with published study showing that reactive astrocytes with enhanced GFAP immunoreactivity 
synthesize more GABA ${ }^{53}$, we also observed a significant increase in GFAP mRNA and protein levels in the auditory cortex of cKO mice (Fig. 7e-f, Supplementary Table 8).

Our data indicate that deletion of Fmrl from astrocytes during postnatal development of inhibitory circuits leads to increased GABA levels in astrocytes, which may trigger a compensatory decrease in synaptic $\mathrm{GABA}_{\mathrm{A}}$ receptors.

\section{Postnatal deletion of $\mathrm{Fmrl}$ in astrocytes leads to increased locomotor activity and decreased socialization in adult cKO mice.}

Cortical hyperexcitability as a result of aberrant PV cell development and impaired inhibition is also observed in several ASD mouse models ${ }^{54}$ and may underlie ASD-like behaviors, such as impaired social behaviors, and enhanced anxiety and hyperactivity. Therefore, we tested adult male Ctrl WT and cKO mice ( $\mathrm{n}=8$ per group) for hyperactivity, anxiety-like behaviors, and sociability in elevated plus maze (Fig. 8a, b), open field (Fig. 8c, d) and threechamber (Fig. 8e, f) tests. cKO mice demonstrated increased locomotor activity by making significantly more total arm entries (Fig. 8a), significantly more line crosses (Fig. 8c) and by showing a significantly higher average speed compared to Ctrl WT mice (Fig. 8a, c, Supplementary Table 9). However, cKO mice showed no changes in anxiety-like behaviors when compared to Ctrl WT mice (Fig. 8b, d). Assessment of sociability and social novelty preference using a three-chamber test showed impaired sociability of adult male cKO mice in session 1 (Fig. 8e, Supplementary Table 10), as well as impaired social novelty preference in session 2 (Fig. 8f, Supplementary Table 10). Our findings establish that postnatal deletion of FMRP from astrocytes leads to increased locomotor activity and impaired socialization in adult mice, while no changes are observed in anxiety-like behaviors. 


\section{Discussion}

Recent studies in human and mouse models of FXS and other ASDs suggest that impaired inhibition, particularly the impaired development of PV-expressing interneurons, underlies cortical hyperexcitability through yet unknown mechanism. The main findings of this study support a novel non-neuronal mechanism of cortical hyperexcitability and demonstrate that the deletion of Fmrl from astrocytes during postnatal developmental period of inhibitory circuit refinements affects (1) GABAergic synapses and GABA levels; (2) PV cell development and connectivity; (3) Neural oscillations and cortical responses to sound; and (4) FXS-associated behaviors including hyperactivity and sociability in adult mice.

First, decreased PV levels as well as a reduction in synaptic $\mathrm{GABA}_{\mathrm{A}} \mathrm{R}$ can be explained by the loss of PV-expressing GABAergic interneurons and/or impaired connectivity between excitatory neurons and PV cells. Indeed, we observed a reduction in the number of PVexpressing cells and excitatory drive onto PV-expressing neurons, as well as lower density of vGAT/Gephyrin perisomatic GABAergic synapses. Abnormal density and function of PV cells is a common finding in FXS and is proposed to underlie cortical hyperexcitability ${ }^{13,21,55}$. Although the mechanism of PV cell dysfunction in astrocyte-specific Fmrl KO is still unclear, recent study suggests that GABA-receptive microglial cells negatively regulate perisomatic PVexpressing inhibitory synapses during the same P15-P30 developmental period in the somatosensory and visual cortex ${ }^{56}$. It is possible that increased levels of astrocytic GABA may trigger excessive pruning of PV-expressing perisomatic synapses in the auditory cortex as well. Moreover, previous studies support the role of Fmrl KO astrocytes in synaptic changes in neurons that can be restored by developmental Fmrl re-expression in astrocytes ${ }^{29}$. 
Second, our findings show that similar to global Fmrl KO mice, loss of Fmrl from astrocytes enhanced baseline gamma power and impaired sound-evoked gamma synchronization in adult $\mathrm{AuC}$ and FC. The deficits were more severe than in forebrain excitatory neuron-specific Fmrl KO mice, which did not show EEG deficits in the frontal cortex ${ }^{14,57}$ suggesting key role of astrocytes in GABAergic dysregulation observed in FXS. It may seem counter-intuitive that reduced PV cell function reduced gamma synchronization while enhancing baseline gamma power. However, this is indeed consistent with recent data suggesting decreased oscillatory and increased broadband gamma power can arise from long-term deficiency in PV inhibition ${ }^{58-61}$. Abnormal sound-evoked gamma synchronization is consistent with the observed reduction in synaptic $\mathrm{GABA}_{\mathrm{A}}$ Rs and PV-positive inhibitory connections as cortical gamma oscillations are linked to the function of PV interneurons ${ }^{58,61,62}$. Our data also show that induced power of alpha and gamma oscillations was higher during sound onset and remained elevated after the sound presentation, supporting dysfunction in inhibitory control of cortical activity. These longerlatency responses quantify oscillatory neural dynamics that extend beyond the onset response, and have been demonstrated in other mammals, including humans ${ }^{63-66}$. The responses quantify stimulus-induced dynamics of neural oscillations and has been associated with cortical activation and attention in sensory cortex ${ }^{67-69}$. Metherate \& Cruikshank (1999) showed that thalamic stimulation caused an early glutamatergic potential in the input layer of auditory cortex, which was followed by gamma-band fluctuations driven by polysynaptic activity in the middle and superficial layers propagated intracortically ${ }^{70}$. This may facilitate formation of transient neural assemblies by synchronizing activity across rapidly changing pools of neurons. The increased gamma band induced power may suggest an abnormal synchronization and enhancement of cortical activation, with implications for sensory hypersensitivity. Our data show that the loss of 
FMRP from astrocytes leads to increased gamma band power of on-set and on-going response to sound, but impaired gamma phase-locking to sound in both auditory and frontal cortices, which may cause sensory discrimination deficits ${ }^{58,61}$ and can lead to cognitive impairments in FXS.

We observed reduced social interactions and increased locomotor activity in astrocytespecific Fmrl cKO mice. Astrocytes are known to modulate cognitive functions and complex animal behaviors, such as emotion, motor activity, memory formation, and sensory processing ${ }^{71}$. It is possible that astrocytic FMRP modulates FXS-related behaviors, especially locomotor activity and social behaviors, through the regulation of PV cell development and functions. Although how astrocytes modulate inhibition remains largely unknown, some progress has been made in defining astrocyte-mediated mechanisms in modulating synaptic connectivity and plasticity in the developing and adult $\mathrm{CNS}^{27,72,73}$. Indeed, alterations of astroglia-secreted synaptogenic signals have been observed in Fmrl KO mice ${ }^{74}$. In addition, our analysis of gene expression in the hippocampus and cortex of astrocyte-specific Fmrl cKO mice has identified several genes that are directly linked to GABAergic synaptic transmission and inhibition.

We found that postnatal Fmrl deletion from astrocytes leads to increased GABA levels in astrocytes but decreased expression of synaptic GABA $\mathrm{R}$ and PV levels. Previous studies have shown reduced expression of astroglial glutamate transporter GLT1 and impaired clearance of extracellular glutamate in astroglial Fmrl cKO mice that may contribute to enhanced hyperexcitability $28,31,75$. However, whether astrocytic $F m r 1$ affects GABA-mediated signaling remains largely unexplored. FMRP is shown to target the mRNAs encoding eight different

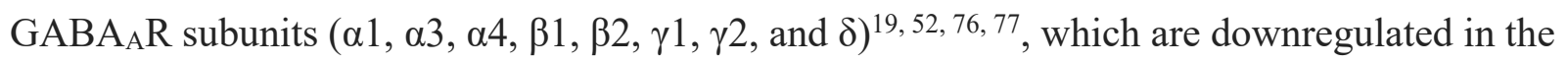
neocortex of Fmrl KO mice ${ }^{16,78,79}$ and humans with $\mathrm{FXS}^{80}$. However, clinical trials using drugs that enhance $\mathrm{GABA}_{\mathrm{A}} \mathrm{R}$ signaling failed to show clinical benefits ${ }^{17,81}$. Our findings demonstrate 
that astrocyte-specific deletion of $F m r l$ also leads to reduced expression of synaptic GABA $\mathrm{R}$ subunits $\alpha 1, \alpha 3$, and $\gamma 2$, indicating that the reduced $\mathrm{GABA}_{\mathrm{A}} \mathrm{R}$ levels cannot be explained by direct interaction between FMRP and $\mathrm{GABA}_{\mathrm{A}} \mathrm{R}$ mRNA in neurons. In contrast, the expression of extrasynaptic $\mathrm{GABA}_{\mathrm{A}}$ receptor subunits $\alpha 5$ and $\delta$ remained unchanged in both hippocampus and cortex of astrocyte-specific Fmrl cKO mice, which is consistent with a study on FX human neurons derived from three different lines of FXS-hESCs that also showed no changes in extrasynaptic $\mathrm{GABA}_{\mathrm{A}} \mathrm{R} \delta$ subunit $^{82}$.

Astrocytes are proposed to be a source of GABA that regulates tonic inhibition ${ }^{83,84}$. Pharmacological blockade of tonic inhibition in hippocampal slices was shown to selectively enhance the excitability of interneurons ${ }^{85}$. Therefore, it is possible that enhanced synthesis and release of GABA from astrocytes may contribute to PV cell hypofunction. Our results showing an increase in the levels of GABA synthesizing enzymes GAD65/67 and GABA levels in Fmr1 KO astrocytes are consistent with published LC-MS/MS proteomics data that show increased protein levels of GAD65/67 in Fmrl KO astrocytes, two of only five proteins that were upregulated in Fmrl KO astrocytes, with no changes in mRNA levels ${ }^{86}$. There is also evidence that translation gad2 gene can be regulated by FMRP through a miR-21-5p mechanism. GABA synthesis from putrescine through the DAO/Adh1a1 pathway can also contribute to the increased GABA levels in astrocytes ${ }^{83}$. Although our results show no changes in Aldha1 protein levels, mRNA levels of Aldha1 and DAO were upregulated in hippocampus and cortex following Fmrl deletion from astrocytes (Supplementary Fig.8). It is still unclear whether the upregulation of astrocytic GABA alters extracellular GABA levels and affects tonic inhibition in the cortex or if there is an alteration in the trafficking of the $\mathrm{GABA}_{\mathrm{A}} \mathrm{R}$ to the membrane surface. Published work shows that tonic GABA-mediated currents are reduced in subiculum of global Fmrl KO mice ${ }^{18}$, 
possibly due to the down-regulation of the $\mathrm{GABA}_{\mathrm{A}} \mathrm{R}$ subunits responsible for tonic inhibition ${ }^{16}$, 78, 79 . Although we see an increase in GABA levels in astrocyte-specific Fmrl cKO mice, previous work showed a decrease in neuronal GABA levels in global Fmrl KO mice ${ }^{17,76}$, suggesting an opposing effects of Fmrl deletion on GABA levels in neurons and astrocytes. While FMRP loss from neurons most likely contributes to the impaired inhibition in FXS, our data suggest that the role of astrocytic GABA should be considered in clinical studies targeting extrasynaptic GABA in FXS, as FMRP may differentially affect GABAergic signaling in neurons and astrocytes.

Together, our findings provide novel insights into the role of astrocytic FMRP in the development of cortical hyperexcitability, suggesting non-neuronal mechanism of abnormal inhibitory circuit development in FXS and calling for a better understanding how astrocytes shape inhibitory responses in the healthy and diseased brain with direct consequences for therapeutic interventions in FXS and other ASDs.

\section{Methods}

\section{Ethics Statement}

All mouse studies were done according to National Institutes of Health and Institutional Animal Care and Use Committee at the University of California Riverside (approval AUP20190015 and AUP20190029) guidelines. All procedures were approved by IACUC; animal welfare assurance \#A3439-01 is on file with the Office of Laboratory Animal Welfare. Mice were maintained in an AAALAC accredited facility under $12 \mathrm{~h}$ light/dark cycle and fed standard mouse chow. Food and water were provided to the mice ad libitum.

\section{Mice}


To achieve specific Fmrl deletion in astrocytes, three different mouse lines were generated. In Group 1, ERT2-Cre ${ }^{G F A P}$ (B6.Cg-Tg(GFAP-cre/ERT2)505Fmv/J

RRID:IMSR JAX:012849) male mice were crossed with $F m r l^{\text {flox/flox }}$ female mice (generated in and obtained from the laboratory of Dr. David Nelson (Baylor College of Medicine, Houston, Texas) ${ }^{87}$ ) to obtain ERT2-Cre ${ }^{G F A P} F m r l^{\text {flox/y }}$ condition KO (cKO) male mice. ERT2-Cre ${ }^{G F A P}$ mice were designated as control WT (Ctrl WT). In Group 2, ERT2-Cre ${ }^{\text {GFAP }}$ mice and ERT2Cre ${ }^{G F A P} F m r 1^{\text {flox/flox }}$ mice were crossed with Rosa-CAG-LSL-tdTomato reporter mice (CAGtdTomato; RRID:IMSR JAX:007909) to generate tdTomatoERT2-Cre ${ }^{\text {GFAP }}$ Ctrl WT and tdTomatoERT2-Cre ${ }^{G F A P} F m r 1^{\text {flox/y }} \mathrm{cKO}$ mice, respectively, allowing for tdTomato expression in astrocytes and analysis of Fmr1 levels. In Group 3, ERT2-Cre ${ }^{G F A P}$ mice and ERT2Cre ${ }^{G F A P} F m r 1^{\text {flox/flox }}$ mice were crossed with Pvalb-tdTomato mice (Tg(PvalbtdTomato)15Gfng/J, RRID:IMSR_JAX:027395) to obtain Pvalb-tdTomato-ERT2-Cre ${ }^{G F A P}$ Ctrl WT and Pvalb-tdvTomato-ERT2-Cre ${ }^{G F A P} F m r 1^{\text {flox/y }} \mathrm{cKO}$ mice, respectively, allowing for tdTomato expression in parvalbumin (PV) inhibitory interneurons for analysis of inhibitory and excitatory synapses. Real-time PCR-based analysis of genomic DNA isolated from mouse tails was used to confirm genotypes by Transnetyx.

In all groups of mice, $\mathrm{Ctrl} \mathrm{WT}$ and $\mathrm{cKO}$ mice received tamoxifen at P14 intraperitoneally ( $0.5 \mathrm{mg}$ in $5 \mathrm{mg} / \mathrm{ml}$ of 1:9 ethanol/sunflower seed oil solution) once a day for 5 consecutive days, and analysis was performed at P28 or P60 (see "Experimental Timeline" in Fig. 1a). Group 1 was used for immunohistochemistry, Western blot, ELISA, qRT-PCR, Nanostring, EEG, and behavioral analysis; Group 2 was used for immunohistochemical analysis of FMRP levels; and Group 3 was used for immunohistochemical analysis of inhibitory and excitatory synapses.

\section{Methods overview}


As Fmrl is expressed in astrocytes during postnatal development ${ }^{29,30}$ and we observed major changes in PV cell development during postnatal day (P)14-P21, we used ERT2-Cre ${ }^{\text {GFAP }}$ line to delete Fmrl from astrocytes during the same P14-P21 period by administering tamoxifen daily from P14 to P19. To confirm deletion of FMRP in astrocytes, we examined expression of FMRP in the cortex of P28 mice using immunostaining, we also analyzed Fmr 1 mRNA levels in the hippocampus and cortex using qRT-PCR. Once deletion was established, all further analysis was performed in either P28 male mice or in adult P60-P70 male mice. At the ages studied here, accelerated hearing loss in the C57bl/6J mice is minimal (Spongr et al., 1997), and because all group comparisons are within-strain and age-matched, hearing loss is unlikely to factor into the phenotypes described ${ }^{102}$.

At P28, we analyzed the effects of astrocyte specific Fmrl deletion on gene expression in the hippocampus and cortex using the NanoString "Glial Profiling Panel” and qRT-PCR.

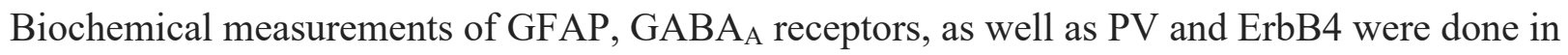
the hippocampus and cortex of cKO and Ctrl WT mice using Western blot. Lastly, immunohistochemistry was used (1) to assess GABA levels in astrocytes, and (2) to analyze excitatory and inhibitory synapses by measuring vGlut1/PSD95 and vGAT/Gephyrin colocalization in the hippocampus and cortex of $\mathrm{cKO}$ and $\mathrm{Ctrl}$ WT mice.

In adult P60-P70 mice, EEG recordings were performed in awake, freely moving mice to determine the long-term effects of postnatal FMRP deletion in astrocytes on neural oscillations in the adult auditory and frontal cortex at baseline and in response to sound ${ }^{37}$. Finally, we used established behavioral tests to examine hyperactivity, anxiety-like behaviors, and socialization in cKO mice.

\section{Primary astrocyte culture}


Primary astrocyte cultures were prepared from P1-3 C57BL/6 (RRID:

IMSR_JAX:003025) littermates WT and KO male mice as described ${ }^{88,89}$ with minor modifications. Briefly, cortex from both hemispheres was dissected, cut into 1-2 mm pieces, placed into a separate tube (cortices of one mouse per tube) and treated with a papain/DNase I (0.1 M PBS/0.1\% BSA/ 25 mM glucose/5\% papain/1×DNase I [Sigma, catalog \#D5025-15K]) solution for $20 \mathrm{~min}$ at $37^{\circ} \mathrm{C}$. Cells were mechanically dissociated and plated on poly-D-lysine (0.05 mg/mL; Sigma, catalog \#P7886) T25 flask (cortices of one mouse per flask) in Dulbecco's Modified Eagle Medium (Gibco, catalog \#11995-065) containing 1\% penicillin-streptomycin (Gibco, catalog \#15140-122) and supplemented with 10\% fetal bovine serum (FBS; Gibco, catalog \# 10438-018) under $10 \% \mathrm{CO}_{2}$ atmosphere at $37^{\circ} \mathrm{C}$. To achieve purified astrocyte cultures ( $>95 \%$ astrocytes) cells were shaken after 4-5 days in vitro (DIV) for $30 \mathrm{~min}$ at $180 \mathrm{rpm}$ (to remove microglia) and then $4 \mathrm{~h}$ at $240 \mathrm{rpm}$ (to remove oligodendrocyte precursor cells). After shaking, the media were removed, and cells were washed twice with $0.1 \mathrm{M} \mathrm{PBS}$ (pH 7.4). Astrocytes were then treated with $0.25 \%$ trypsin/EDTA solution (Gibco, catalog \#25200-056) for 2 min at $37^{\circ} \mathrm{C}$ and plated on poly-D-lysine coated T75 flask in DMEM containing $10 \% \mathrm{FBS}$. Once confluent, astrocytes were trypsinized and plated on coverslips in 24-well plates at a density of 35,000 cells per well and T75 flask (all remaining cells). Astrocytes were cultured for 2 days before being fixed for immunocytochemistry (in 24-well plate) or until confluent for Western blot (in T75 flask). For immunostaining, astrocytes were fixed with $2 \%$ paraformaldehyde for $30 \mathrm{~min}$ at room temperature, washed three times with PBS $0.1 \mathrm{M}$ and stained as described below. For Western blot, cells were collected from T75 flask and lysed in the lysis buffer $(25 \mathrm{mM}$ Tris- $\mathrm{HCl}, 150 \mathrm{mM} \mathrm{NaCl}, 5 \mathrm{mM}$ EDTA, 1\% Triton X-100, $1 \mathrm{mM}$ sodium pervanadate, and protease inhibitor cocktail [1:100, Sigma, catalog \#P8340]) at $4^{\circ} \mathrm{C}$ for $30 \mathrm{~min}$. 
Cell lysates were cleared by centrifugation at $13,500 \mathrm{rpm}$ for $20 \mathrm{~min}$ at $4^{\circ} \mathrm{C}$. Samples were further concentrated using protein concentrators (Pierce, catalog \#88513) to achieve 10x concentration and processed for Western blot.

\section{Immunofluorescence}

P28 male Ctrl WT and cKO mice were euthanized with isoflurane and sodium pentobarbital and perfused transcardially first with cold phosphate-buffered saline (PBS, $0.1 \mathrm{M})$ to clear out the blood and then with $4 \%$ paraformaldehyde (PFA) in $0.1 \mathrm{M}$ PBS for fixation. Brains were removed and post-fixed for $2-4 \mathrm{~h}$ in $4 \%$ PFA. $40-100 \mu \mathrm{m}$ brain slices were obtained using a vibratome (5100 mz Campden Instruments). Hippocampus, auditory cortex (AuC), and frontal cortex (FC) were identified using the brain atlas ${ }^{90}$. For each brain, an average of 5-6 brain slices were collected per region.

Immunostaining for FMRP was performed using antigen retrieval methods, as previously described $^{14}$. Briefly, $40 \mu \mathrm{m}$ brain slices obtained from tdTomatoERT2-Cre ${ }^{G F A P} F m r l^{\text {flox/y }} \mathrm{cKO}$ and tdTomatoERT2-Cre ${ }^{G F A P} \mathrm{Ctrl}$ WT male mice were stained overnight with mouse anti-FMRP (1:100; Developmental Studies Hybridoma Bank, catalog \#2F5-1-s, RRID: AB_10805421). Secondary antibody was donkey anti-mouse Alexa 488 (4 $\mu \mathrm{g} / \mathrm{mL}$; Molecular Probes, catalog\# A21202, RRID: AB_141607). Slices were mounted on slides with Vectashield mounting medium containing DAPI (Vector Laboratories, catalog \# H-1200, RRID:AB_2336790) and Cytoseal.

Immunostaining in $100 \mu \mathrm{m}$ brain slices containing hippocampus, auditory cortex, and frontal cortex was performed as previously described ${ }^{14}$. For slices obtained from ERT2-

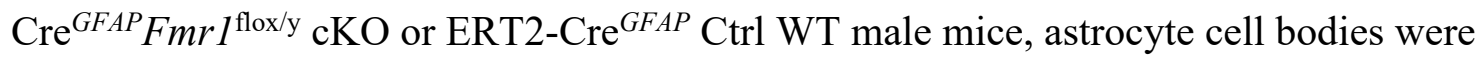
identified by immunolabeling against Glutamine Synthetase using rabbit anti-Glutamine Synthetase antibody (1:500, Sigma-Aldrich catalog\# G2781, RRID:AB_259853), and GABA 
immunoreactivity was detected by immunostaining with guinea pig anti-GABA antibody (1:500, Abcam, catalog \# ab17413, RRID:AB_443865). For slices obtained from Pvalb-tdvTomatoERT2-Cre ${ }^{G F A P} F m r 1^{\text {flox/y }}$ cKO and Pvalb-tdTomato-ERT2-Cre ${ }^{G F A P}$ Ctrl WT male mice, excitatory presynaptic boutons were labeled by immunostaining against vGlut1 using rabbit anti-vGlut1 antibody (0.25 mg/ml, Invitrogen, catalog \# 482400, RRID:AB_2533843), and excitatory postsynaptic sites were identified with mouse anti-PSD95 antibody $(1.65 \mu \mathrm{g} / \mathrm{ml}$, Invitrogen, catalog \# MA1-045, RRID:AB_325399). Inhibitory presynaptic sites were detected by immunolabeling against vesicular GABA transporter (vGAT) using rabbit anti-vGAT antibody (1:100, Synaptic Systems, catalog \# 131002, RRID:AB_887871). Inhibitory postsynaptic sites were detected by immunolabeling against gephyrin using mouse anti-gephyrin antibody (1:500, Synaptic Systems, catalog \# 147111, RRID:AB_887719). Secondary antibodies used were as follows: AlexaFluor-647-conjugated donkey anti-rabbit IgG (4 $\mu \mathrm{g} / \mathrm{ml}$, Invitrogen, catalog \# A31573, RRID:AB_2536183), AlexaFluor-488-conjugated goat anti-guinea pig IgG (4 $\mu \mathrm{g} / \mathrm{ml}$, Invitrogen, catalog \# A-11073, RRID:AB_2534117), AlexaFluor-488-conjugated goat antimouse IgG (4 g/ml, Invitrogen, catalog \# A-11001, RRID:AB_2534069), AlexaFluor-488conjugated donkey anti-rabbit IgG (4 $\mu \mathrm{g} / \mathrm{ml}$, Invitrogen, A-21206, RRID:AB_2535792), and AlexaFluor-647-conjugated donkey anti-mouse IgG (4 $\mu \mathrm{g} / \mathrm{ml}$, Invitrogen, catalog \# A-31571, RRID:AB_162542). Slices were mounted on slides with Vectashield mounting medium containing DAPI (Vector Laboratories, catalog \# H-1200, RRID:AB_2336790) and Cytoseal. Immunostaining of fixed primary astrocytes was performed as follows. Cells were blocked for $1 \mathrm{~h}$ at room temperature with 5\% NDS in $0.1 \%$ Triton X-100 in $0.1 \mathrm{M}$ PBS an then immunolabeled with guinea pig anti-GABA antibody (1:1000, Abcam, catalog \# ab17413, RRID:AB_443865) and rabbit anti-GS (1:1000, Sigma-Aldrich Cat\# G2781, RRID:AB_259853) 
antibody in blocking buffer overnight at $4^{\circ} \mathrm{C}$. Coverslips then were washed $3 \times 10$ min with $1 \%$ NDS in 0.1 M PBS at room temperature followed by the incubation with goat anti-guinea pig Alexa Fluor 488 (4 g/ml, Invitrogen, catalog \#A-11073, RRID:AB_2534117) and donkey antirabbit Alexa Fluor 594 (4 $\mu \mathrm{g} / \mathrm{ml}$, Invitrogen, catalog \#A-21207, RRID:AB_141637) for $2 \mathrm{~h}$ at room temperature. Coverslips were washed $3 \times 10$ min with $0.1 \mathrm{M}$ PBS, mounted on slides with Vectashield mounting medium containing DAPI (Vector Laboratories, \#H1200) and sealed with Cytoseal.

\section{Image Analysis}

Confocal images of coronal brain slices containing CA1 hippocampus, superficial layers of $\mathrm{AuC}$ and $\mathrm{FC}$ were taken with an SP5 confocal laser-scanning microscope (Leica Microsystems) as previously described with modifications ${ }^{73,91}$. Briefly, high-resolution optical sections $(1024 \times 1024$ pixel format $)$ were captured with a $40 \times$ water-immersion and $1 \times$ zoom at $1 \mu \mathrm{m}$ step intervals to assess FMRP immunoreactivity. Confocal images of synaptic puncta were taken using a $40 \times$ objective $(1.2 \mathrm{NA})$, and $1 \times$ zoom at high resolution $(1024 \times 1024$ pixel format $)$ with a $0.5 \mu \mathrm{m}$ intervals. For GABA analysis in primary astrocyte cultures, images were captured using a Nikon Eclipse TE2000-U inverted fluorescent microscope with a $20 \times$ air objective and a Hamamatsu ORCA-AG 12-bit CCD camera using Image-Pro software. For analysis, 20 images were collected (10 images per coverslip, 2 coverslips/animal, 5-6 animals per group) per animal. All images were acquired under identical conditions and processed for analysis as follows: (1) For analysis of overall FMRP immunoreactivity, superficial layers of AuC were analyzed per each brain slice from at least 3 animals per group. Cortical layers were identified as previously reported $^{92}$ and used for layer-specific analysis. Image analysis was performed using ImageJ macro plugin PIPSQUEAK (https://labs.wsu.edu/sorg/research-resources/). 10 images in the Z- 
stack (1.194 pixels $/ \mu \mathrm{m})$ were compiled into a single image using ImageJ macro plugin PIPSQUEAK, scaled, and converted into 32-bit, grayscale, TIFF files. PIPSQUEAK was run in "semi-automatic mode" to select ROIs to identify individual FMRP-positive cells, which were then verified by a trained experimenter. Distributions of density and intensity were compared between experimental groups, to assess differences in FMRP cell density and intensity between Ctrl WT and cKO mice. (2) For analysis of FMRP immunoreactivity in astrocytes, astrocytes were visualized with tdTomato. Cell areas were outlined using selection tool, then cell area, integrated fluorescent intensity, and mean intensity were measured for FMRP in each astrocyte. (3) For analysis of GABA immunoreactivity in Glutamine Synthetase (GS) labeled astrocytes, astrocytic cell bodies in CA1 hippocampus, superficial layers of AuC, FC and primary cultured astrocytes were selected and outlined using selection tool. These ROIs were saved in the ROI manager and used to measure area and perform analysis of integrated fluorescent intensity and mean intensity of GABA in each astrocyte. (4) For the analysis of vGlut1/PSD95 co-localization onto PV cells, three-dimensional fluorescent images were created by the projection of each z stack containing 40-50 high-resolution optical serial sections $(1024 \times 1024$ pixel format $)$ taken at $0.224 \mu \mathrm{m}$ intervals in the X-Y plane. td-Tomato labeled PV somas were selected for the analysis, then PSD95 and vGlut1 puncta (within $1 \mu \mathrm{m}$ distance) of PV soma were selected. Quantifications of vGlut/PSD95 co-localization on enclosed volume of PV cells $\left(\mu \mathrm{m}^{3}\right)$ were conducted using Neurolucida 360 software (MicroBrightField RRID:SCR_001775). (5) For the analysis of vGAT and Gephyrin, vGAT puncta were co-localized with td-Tomato labeled PVpositive pre-synaptic boutons (within $0.5 \mu \mathrm{m}$ distance). PV/vGAT puncta were then co-localized with Gephyrin puncta (within $1 \mu \mathrm{m}$ distance). Quantification of vGAT/Gephyrin, PV/Gephyrin, and vGAT/PV puncta co-localization was conducted using Neurolucida 360 software 
(MicroBrightField RRID:SCR_001775). Statistical analysis was performed with unpaired $t$ test using GraphPad Prism 7 software (RRID:SCR_002798). Data represent mean \pm SEM.

\section{RNA Extraction}

Total RNA was extracted from the hippocampus and cortical regions of Ctrl WT and cKO mice ( $\mathrm{n}=4$ per group) using the RNeasy kit (Qiagen, Valencia, CA, USA) method (see location of cortical areas 1 and 2 in the Supplementary Figure 1). First-strand complementary DNA (cDNA) was generated using iScript ${ }^{\mathrm{TM}}$ cDNA Synthesis Kit (Catalog \#1708891, Bio-Rad), and reverse transcription of total RNA (500 ng) was performed for $26 \mathrm{~min}$ at $37^{\circ} \mathrm{C}$. All surfaces for tissue collection and processing were sanitized using $70 \%$ ethanol and then treated with an RNAse inhibitor (RNAse out, G-Biosciences, St. Louis, MO, USA) to maintain integrity of isolated RNA. cDNA obtained from the hippocampus was used for NanoString analysis, and cDNA from all regions was utilized for qRT-PCR.

\section{NanoString nCounter Gene Expression Assays and Analysis}

nCounter gene expression assays (NanoString Technologies) were performed for the Glial Profiling NanoString Panel. Briefly, panel codeset probes were hybridized with $150 \mathrm{ng}$ of total RNA per hippocampus sample over $18 \mathrm{~h}$ at $65^{\circ} \mathrm{C}$ according to NanoString protocol. Hybridized RNA was then diluted in molecular grade water and loaded into nCounter SPRINT cartridge (NanoString), placed into nCounter SPRINT Profiler, and quantified. RNA-conjugated probes were counted via NanoString Sprint Profiler technology.

nSolver and Advanced Analysis

Results from the panel were normalized in nSolver following best practices, and analyzed using nSolver and Advanced Analysis software according to previously published protocols ${ }^{93,94}$. nSolver-generated heat maps were created using normalized data and agglomerative clustering, a 
bottom-up form of hierarchical clustering (NanoString User Manual C0019-08). For Advanced Analysis, normalized data was used (NanoString User Manual 10030-03). Differential expression (DE) analysis was performed to identify specific targets that exhibit significantly increased or decreased expression compared to Ctrl WT values. Gene set analysis was run to determine the change in direction of regulation within each pre-defined gene set relative to Ctrl WT. Global significance scores, a summary T-statistic used to measure change (NanoString User Manual 10030-03) were calculated, and the directed global significance scores were expressed via heatmap. Pathway analysis was also conducted to determine overall changes in pathways based on the first principal component of the targets within a pathway as annotated by NanoString (NanoString User Manual 10030-03). Direction of pathway change (up- or downregulated) was determined by cross referencing the pathway score with the corresponding volcano plot for that pathway. Summary pathway score plot colors are based on calculated scores and are represented as downregulation (yellow) to upregulation (blue).

\section{Separate Statistical Analyses}

Normalized linear counts for all genes in the panel were used in fold change analysis of cKO genes compared to Ctrl WT. Differentially expressed genes (DEGs) were defined by a pvalue $<0.05$. Statistical analysis was performed with unpaired Welch's t-test using GraphPad Prism 7 software (RRID:SCR_002798). Data represent mean \pm standard error of the mean (SEM). Gene enrichment and functional annotation analyses of DEGs was performed using open-source functional enrichment tool DAVID (https://david.ncifcrf.gov/home.jsp). All raw NanoString data are available on:

https://www.dropbox.com/sh/19b420pr1nl1zlh/AAAJ_qe0uhyljeNzFAwVECFsa?dl=0.

\section{Quantitative Real Time PCR Analysis}


qRT-PCR was carried out using PrimePCR Sybr Green Assays (Biorad, Hercules, CA, USA) with the following primers for mouse genes: Fmr1, Gabra1, Gabra3, Gabrg2, Gabra4, Gabra5, Gabrd, Aldhla1, DAO, GFAP, PV, ErbB4, NRG-3, and TrkB (see Supplementary

Table 10 for sequences of primers used for qRT-PCR). GAPDH was selected as a housekeeping gene and no changes in its expression were found across genotype. Relative quantification using the delta-delta $\left(2^{-\Delta \Delta \mathrm{Cq}}\right)$ method was used to compare changes in gene expression between $\mathrm{cKO}$ mice and Ctrl WT mice. Reactions were run in duplicate for each animal. Statistical analysis was performed with unpaired t-test using GraphPad Prism 7 software (RRID:SCR_002798). Data represent mean \pm standard error of the mean (SEM).

\section{Western Blot Analysis}

The hippocampus and cortical regions (see location of cortical areas 1 and 2 in the Supplementary Figure 1) were removed from each mouse ( $\mathrm{n}=4$ mice per group), cooled in PBS, and homogenized in ice-cold lysis buffer (50 mM Tris-HCl, pH 7.4, $150 \mathrm{mM} \mathrm{NaCl,} 5 \mathrm{mM}$ EDTA, $0.05 \%$ Triton X-100, and $1 \mathrm{mM} \mathrm{PMSF)} \mathrm{containing} \mathrm{protease} \mathrm{inhibitor} \mathrm{cocktail} \mathrm{(Sigma,}$ catalog \# P8340) and phosphatase inhibitor cocktail (Sigma, catalog \# P0044). The samples were processed as previously described with modifications ${ }^{14}$. The samples were rotated at $4{ }^{\circ} \mathrm{C}$ for at least $1 \mathrm{~h}$ to allow for complete cell lysis and then cleared by centrifugation at 13,200 rpm for 15 $\min$ at $4^{\circ} \mathrm{C}$. Supernatants were isolated and boiled in reducing sample buffer (Laemmli $2 \times$ concentrate, catalog \# S3401, Sigma), and separated on 8-16\% Tris-Glycine SDS-PAGE precast gels (catalog \# EC6045BOX, Life Technologies). Proteins were transferred onto Protran BA 85 Nitrocellulose membrane (GE Healthcare) and blocked for $1 \mathrm{~h}$ at room temperature in 5\% skim milk (Catalog \#170-6404, Bio-Rad). Primary antibody incubations were performed overnight at $4^{\circ} \mathrm{C}$ with antibodies diluted in TBS $/ 0.1 \%$ Tween-20/5\% BSA. The following primary antibodies 
were: rabbit anti-GABRG2 (1:1000; ThermoFisher Scientific, catalog \#14104-1-AP, RRID:AB_10693527); rabbit anti-GABRA5 (1:1000; ThermoFisher Scientific, catalog \#PA531163, RRID:AB_2548637); rabbit anti-GFAP (1:1000; Cell Signaling, catalog \#12389, RRID:AB_2631098); mouse anti-PV (1:1000; Millipore, catalog \#MAB1572, RRID: AB_2174013); rabbit anti-ErbB4 (1:1000; Cell Signaling, catalog \#4795, RRID:AB_2099883); and rabbit anti-ßactin (1:2000; Abcam, catalog \#ab8227, RRID: AB_2305186).

Blots were washed $3 \times 10$ min with TBS/ $0.1 \%$ Tween-20 and incubated with the appropriate HRP-conjugated secondary antibodies for $1 \mathrm{~h}$ at room temperature in a TBS/0.1\% Tween-20/5\% BSA solution. The secondary antibodies used were HRP-conjugated donkey antimouse IgG (Jackson ImmunoResearch, catalog \#715-035-150, RRID: AB_2340770) or HRPconjugated goat anti-rabbit IgG (Jackson ImmunoResearch, catalog \#111-035-003, RRID: AB_2313567). After secondary antibody incubations, blots were washed $3 \times 10 \mathrm{~min}$ in TBS/0.1\% Tween-20, incubated in ECL 2 Western Blotting Substrate (Thermo Scientific, catalog \#80196) and imaged using ChemiDoc imaging system (Bio-Rad, RRID:SCR_019037). For re-probing, membrane blots were washed in stripping buffer $(2 \%$ SDS, $100 \mathrm{mM} \beta-$ mercaptoethanol, $50 \mathrm{mM}$ Tris- $\mathrm{HCl}, \mathrm{pH} 6.8$ ) for $30 \mathrm{~min}$ at $55^{\circ} \mathrm{C}$, then rinsed repeatedly with TBS/0.1\% Tween-20, finally blocked with 5\% skim milk, and then re-probed. The first set of blots were stained for PV, B-actin, and ErbB4. The second set of blots were probed for GABRG2, GABRA5, GFAP, and $\beta$-actin. Astrocyte cell culture samples were probed for glutamic acid decarboxylase (GAD) 65-67 using rabbit anti-GAD 65-67 (Abcam, catalog \#183999) with the corresponding secondary HRP-conjugated antibody and re-probed for $\beta$-actin (as described above). Band density was analyzed by measuring band and background intensity using Adobe Photoshop CS5.1 software (RRID:SCR_014199). 3-4 samples per group (Ctrl WT 
vs. cKO or Ctrl WT vs KO for astrocyte cultures) were run per blot, and precision/tolerance $(\mathrm{P} / \mathrm{T})$ ratios for individual $\mathrm{cKO}$ samples were normalized to $\mathrm{P} / \mathrm{T}$ ratios of $\mathrm{Ctrl} \mathrm{WT}$ samples with the similar actin levels. Statistical analysis was performed with unpaired t-test using GraphPad Prism 7 software (RRID:SCR 002798). Data represent mean \pm standard error of the mean (SEM).

\section{Surgery for in vivo EEG recordings}

Age-matched adult P60-70 male Ctrl WT $(n=10)$ and cKO $(n=9)$ mice were used for the EEG studies as previously described with modifications ${ }^{14,37}$. Mice were anesthetized with isoflurane inhalation (0.2-0.5\%) and an injection of ketamine and xylazine (K/X) (i.p. 80/10 $\mathrm{mg} / \mathrm{kg}$ ), and then secured in a bite bar, and placed in a stereotaxic apparatus (model 930; Kopf, CA). Artificial tear gel was applied to the eyes to prevent drying. Toe pinch reflex was used to measure anesthetic state every 10 min throughout the surgery, and supplemental doses of $\mathrm{K} / \mathrm{X}$ were administered as needed. Once the mouse was anesthetized, a midline sagittal incision was made along the scalp to expose the skull. A Foredom dental drill was used to drill $1 \mathrm{~mm}$ diameter holes in the skull overlying the right auditory cortex $(-1.6 \mathrm{~mm},+4.8 \mathrm{~mm})$, left frontal lobe $(+3.0$ $\mathrm{mm},-1.6 \mathrm{~mm})$, and left occipital $(-4.2 \mathrm{~mm},-5.1 \mathrm{~mm})$ (coordinate relative to Bregma: anterior/posterior, medial/lateral). Three channel electrode posts from Plastics One (MS333-2-ASPC) were attached to $1 \mathrm{~mm}$ stainless steel screws from Plastics One (8L003905201F) and screws were advanced into drilled holes until secure. Special care was taken not to advance the screws beyond the point of contact with the Dura. Dental cement was applied around the screws, on the base of the post, and exposed skull. Triple antibiotic was applied along the edges of the dental cement followed by an injection of subcutaneous Buprenorphine $(0.1 \mathrm{mg} / \mathrm{kg})$. Mice were placed on a heating pad to aid recovery from anesthesia. A second Buprenorphine injection was 
administered between 6 and 10 hours after surgery. Mice were then individually housed, returned to the vivarium and monitored daily until the day of EEG recordings. The separation between the last post-surgical Buprenorphine injection and EEG recordings was between 3 and 5 days.

\section{Electrophysiology}

Baseline and auditory event-related potential (ERP) recordings were obtained using the BioPac system (BIOPAC Systems, Inc.) from awake and freely moving mice as published previously with modifications ${ }^{37}$. Mice were allowed to habituate to the recording chamber for 15 min prior to being connected to the BioPac system. A three-channel tether was connected to the electrode post (implanted during surgery) under brief isoflurane anesthesia. The mouse was then placed inside a grounded Faraday cage after recovery from isoflurane. This tether was then connected to a commutator located directly above the cage. Mice were then allowed to habituate while being connected to the tether for an additional 20 min before EEG recordings were obtained.

The BioPac MP150 acquisition system was connected to two EEG 100C amplifier units (one for each channel) to which the commutator was attached. The lead to the occipital cortex was used as reference for both frontal and auditory cortex screw electrodes. The acquisition hardware was set to high-pass $(>0.5 \mathrm{~Hz})$ and low-pass $(<100 \mathrm{~Hz})$ filters. Normal EEG output data were collected with gain maintained the same $(10,000 \mathrm{x})$ between all recordings. Data were sampled at a rate of either 2.5 or $5 \mathrm{kHz}$ using Acqknowledge software and down sampled to $1024 \mathrm{~Hz}$ post hoc using Analyzer 2.1 (Brain Vision Inc.). Sound delivery was synchronized with EEG recording using a TTL pulse to mark the onset of each sound in a train. Baseline EEGs were recorded for $5 \mathrm{~min}$ (no auditory stimuli were presented), followed by recordings in 
response to auditory stimulation. After these experiments were completed, mice were returned to the colony and euthanized on a later date.

\section{Acoustic Stimulation}

All experiments were conducted in a sound-attenuated chamber lined with anechoic foam (Gretch-Ken Industries, OR) as previously described with modifications ${ }^{14,37}$. Acoustic stimuli were generated using RVPDX software and RZ6 hardware (Tucker-Davis Technologies, FL) and presented through a free-field speaker (MF1 Multi-Field Magnetic Speaker; Tucker-Davis Technologies, FL) located 12 inches directly above the cage. Sound pressure level (SPL) was modified using programmable attenuators in the RZ6 system. The speaker output was $\sim 65-70 \mathrm{~dB}$ SPL at the floor of the recording chamber with fluctuation of $+/-3 \mathrm{~dB}$ for frequencies between 5 and $35 \mathrm{kHz}$ as measured with a $1 / 4$ inch Bruel \& Kjaer microphone.

We used acoustic stimulation paradigms that have been previously established in Fmrl $\mathrm{KO}$ mice ${ }^{37}$, which is analogous to work in humans with $\mathrm{FXS}^{47}$. A chirp-modulated signal (henceforth, 'chirp') to induce synchronized oscillations in EEG recordings was used to measure temporal response fidelity. The chirp is a $2 \mathrm{~s}$ broadband noise stimulus with amplitude modulated (100\% modulation depth) by a sinusoid whose frequencies increase (Up-chirp) or decrease (Down-chirp) linearly in the $1-100 \mathrm{~Hz}$ range ${ }^{95-97}$. The chirp facilitates a rapid measurement of transient oscillatory response (delta to gamma frequency range) to auditory stimuli of varying frequencies and can be used to compare oscillatory responses in different groups in clinical and pre-clinical settings ${ }^{97}$. Inter-trial coherence analysis ${ }^{69}$ can then be used to determine the ability of the neural generator to synchronize oscillations to the frequencies present in the stimulus. 
To avoid onset responses contaminating phase locking to the amplitude modulation of the chirp, the stimulus was ramped in sound level from $0-100 \%$ over $1 \mathrm{~s}$ (rise time), which then smoothly transitioned into chirp modulation of the noise. Up and Down chirp trains were presented 300 times each (for a total of 600 trains). Both directions of modulation were tested to ensure any frequency specific effects were not due to the frequency transition history within the stimulus. Up- and Down- chirp trains were presented in an alternating sequence. The interval between each train was randomly varied between 1 and $1.5 \mathrm{~s}$.

To study evoked response amplitudes and habituation, trains of $100 \mathrm{~ms}$ broadband noise were presented at two repetition rates, $0.25 \mathrm{~Hz}$ (a non-habituating rate) and $4 \mathrm{~Hz}$ (a strongly habituating rate $)^{48}$. Each train consisted of 10 noise bursts and the inter-train interval used was 8 seconds. Each repetition rate was presented 100 times in an alternating pattern ${ }^{48}$. The onset of trains and individual noise bursts were tracked with separate TTL pulses that were used to quantify latency of response.

\section{EEG Data Analysis}

Data were extracted from Acqknowledge and files saved in a file format (EDF) compatible with BrainVision Analyzer 2.1 software as previously described with modifications ${ }^{14}$ 37. All data were notch filtered at $60 \mathrm{~Hz}$ to remove residual line frequency power from recordings. EEG artifacts were removed using a semi-automatic procedure in Analyzer 2.1 for all recordings. Less than $20 \%$ of data were rejected due to artifacts from any single mouse. Baseline EEG data were divided into $2 \mathrm{~s}$ segments and Fast Fourier Transforms (FFT) were calculated on each segment using $0.5 \mathrm{~Hz}$ bins and then average power $\left(\mu \mathrm{V}^{2} / \mathrm{Hz}\right)$ was calculated for each mouse from 1-100 Hz. Power was then binned into standard frequency bands: Delta (1-4 Hz), Theta (4$10 \mathrm{~Hz})$, Alpha (10-13 Hz), Beta (13-30 Hz), Low Gamma (30-55 Hz), and High Gamma (65-100 
Hz). Responses to chirp trains were analyzed using Morlet wavelet analysis. Chirp trains were segmented into windows of $500 \mathrm{~ms}$ before chirp onset to $500 \mathrm{~ms}$ after the end of the chirp sound (total of $3 \mathrm{~s}$ because each chirp was $2 \mathrm{~s}$ in duration). EEG traces were processed with Morlet wavelets from 1-100 $\mathrm{Hz}$ using complex number output (voltage density, $\mu \mathrm{V} / \mathrm{Hz}$ ) for ITPC calculations, and power density $\left(\mu \mathrm{V}^{2} / \mathrm{Hz}\right)$ for non-phase locked single trial power (STP) calculations and baseline corrected non-phase locked single trial power (induced power). Wavelets were run with a Morlet parameter of 10 as this gave the best frequency/power discrimination. This parameter was chosen since studies in humans found most robust difference around $40 \mathrm{~Hz}$, where this parameter is centered ${ }^{47}$. To measure phase synchronization at each frequency across trials Inter Trial Phase Coherence (ITPC) was calculated. The equation used to calculate ITPC is:

$$
\operatorname{ITPC}(f, t)=\frac{1}{n} \sum_{k=1}^{n} \frac{F_{k}(f, t)}{\left|F_{k}(f, t)\right|}
$$

where $f$ is the frequency, $t$ is the time point, and $k$ is trial number. Thus, $F_{k}(f, t)$ refers to the complex wavelet coefficient at a given frequency and time for the $k$ th trial. There were no less than 275 trials (out of 300) for any given mouse after segments containing artifacts were rejected. All raw EEG data analysis is available on:

\section{https://www.dropbox.com/sh/kerg6hj3kcnon81/AABrMHa2BW8Rwckng8e9wZ9ba?dl=0}

\section{EEG Statistical Analysis}

Statistical group comparisons of chirp responses (ITPC and STP) and broadband noise trains (ITPC and induced power) were quantified by wavelet analysis. Analysis was conducted by binning time into 256 parts and frequency into 100 parts, resulting in a 100x256 matrix. Nonparametric cluster analysis was used to determine contiguous regions in the matrix that were 
significantly different from a distribution of 1000 randomized Monte Carlo permutations based on previously published methods with modifications ${ }^{98}$. Briefly, if the cluster sizes of the real genotype assignments (both positive and negative direction, resulting in a two-tailed alpha of $\mathrm{p}=$ 0.025) were larger than $97.25 \%$ of the random group assignments, those clusters were considered significantly different between genotypes. This method avoids statistical assumptions about the data and corrects for multiple comparisons.

Because movement can alter cortical gain ${ }^{99,100}$, and Fmrl KO mice show hyperactivity, a piezoelectric transducer was placed underneath the recording cage to detect when the mouse was moving. The term 'baseline' is used to indicate EEGs recorded in these mice without any specific auditory stimuli. In all cases where genotype means are reported, SEM was used. The genotype differences in baseline power were analyzed on 6 dependent variables using one-way Multivariate analysis of co-variance (MANCOVA) with one covariate (movement), Independent Variables (IV): Genotype (Ctrl WT and cKO mice), dependent variables (DV): 6 frequency bins (delta to high gamma). The proportion of time spent moving during the 5-minute recording session was used as a covariate to isolate effects of genotype and control for the effect movement has on cortical gain. When multiple comparisons for MANCOVA were made, genotype comparisons were corrected using Bonferroni adjustments. The divisor for Bonferroni correction for multiple comparisons (for 6 frequency bands) on MANCOVA was set to $6, a=0.05 / 6=$ 0.0083. Data are often expressed and plotted as ratio of control group values to gauge relative differences in various factors using the same scale.

\section{Behavioral Assessments}

Social Novelty Test 
Sociability and social memory were studied in age-matched adult Ctrl WT and cKO mice ( $\mathrm{n}=8$ per group) using a three-chamber test as described previously with minor modifications ${ }^{73}$. Briefly, a rectangular box contained three adjacent chambers $19 \times 45 \mathrm{~cm}$ each, with 30 -cm-high walls and a bottom constructed from clear Plexiglas. The three chambers were separated by dividing walls, which were made from clear Plexiglas with openings between the middle chamber and each side chamber. Removable doors over these openings permitted chamber isolation or free access to all chambers. All testing was done in a brightly lit room (650 lux), between 9:00 A.M. and 2:00 P.M. Before testing, mice were housed in a room with a $12 \mathrm{~h}$ light/dark cycle with ad libitum access to food and water. The cages were transferred to the behavioral room $30 \mathrm{~min}$ before the first trial began for habituation. The test mouse was placed in the central chamber with no access to the left and right chambers and allowed to habituate to the test chamber for $5 \mathrm{~min}$ before testing began. Session 1 measured sociability. In session 1 , another mouse (Stranger 1) was placed in a wire cup-like container in one of the side chambers. The opposite side had an empty cup of the same design. The doors between the chambers were removed, and the test mouse was allowed to explore all three chambers freely for 10 min, while being digitally recorded from above. The following parameters were monitored: the duration of direct contact between the test mouse and either the stranger mouse or empty cup and the duration of time spent in each chamber. Session 2 measured social memory and social novelty preference. In session 2, a new mouse (Stranger 2) was placed in the empty wire cup in the second side chamber. Stranger 1, a now familiar mouse, remained in the first side chamber. The test mouse was allowed to freely explore all three chambers for another $10 \mathrm{~min}$, while being recorded, and the same parameters were monitored. Placement of Stranger 1 in the left or right side of the chamber was randomly altered between trials. The floor of the chamber was cleaned 
with 2\%-3\% acetic acid, $70 \%$ ethanol, and water between tests to eliminate odor trails.

Assessments of the digital recordings were done using TopScan Lite software (Clever Sys., Inc., VA). To measure changes in sociability and social memory, percent time spent in each chamber was calculated in each test. Further, a sociability index

$=\left(\frac{\text { time in } S 1 \text { chamber }}{\text { time in S1 chamber }+ \text { time in empty chamber }}\right)$ and social novelty preference index $=\left(\frac{\text { time in } S 2 \text { chamber }}{\text { time in } S 2 \text { chamber }+ \text { time in } S 1 \text { chamber }}\right)$ were calculated as described previously ${ }^{73,101}$. For sociability index, values $<0.5$ indicate more time spent in the empty chamber, $>0.5$ indicate more time spent in the chamber containing Stranger 1 , and 0.5 indicates equal amount of time in both chambers. For social novelty preference index, values $<0.5$ indicate more time spent in the chamber containing Stranger 1 or now familiar mouse, $>0.5$ indicate more time spent in the chamber containing Stranger 2 or new stranger mouse, and 0.5 indicates equal amount of time in both chambers. Statistical analysis for time spent in each chamber was performed using two-way ANOVA followed by Bonferroni multiple comparison post-test, while statistical analysis for sociability index and social novelty preference index was performed with unpaired t-test using GraphPad Prism 7 software (RRID:SCR_002798). Data represent mean \pm standard error of the mean (SEM).

Open-field test

Anxiety-like behaviors and locomotor activity were tested in age-matched adult Ctrl WT and $\mathrm{cKO}$ mice ( $\mathrm{n}=8$ per group) as described previously with modifications ${ }^{14}$. A $72 \times 72-\mathrm{cm}$ open-field arena with 50-cm-high walls was constructed from opaque acrylic sheets with a clear acrylic sheet for the bottom. The open field arena was placed in a brightly lit room, and one mouse at a time was placed in a corner of the open field and allowed to explore for 10 min while 
being recorded with digital video from above. The floor was cleaned with 2-3\% acetic acid, 70\% ethanol, and water between tests to eliminate odor trails. The mice were tested between the hours of 9:00 A.M. and 1:00 P.M., and this test was always performed prior to the elevated plus maze. The arena was subdivided into a $4 \times 4$ grid of squares with the middle of the grid defined as the center. A line $4 \mathrm{~cm}$ from each wall was added to measure thigmotaxis. Locomotor activity was scored by the analysis of total line crosses and speed using TopScan Lite software (Clever Sys., Inc., VA). A tendency to travel to the center (total number of entries into large and small center squares) and the time in thigmotaxis were used as an indicator of anxiety. The analysis was performed in 5 min intervals for the total $10 \mathrm{~min}$ exploration duration. Assessments of the digital recordings were performed blind to the condition. Statistical analysis was performed with unpaired t-test using GraphPad Prism 7 software (RRID:SCR_002798). Data represent mean \pm standard error of the mean (SEM).

\section{Elevated plus maze}

The elevated plus maze consisted of four arms in a plus configuration. Two opposing arms had 15-cm tall walls (closed arms), and two arms were without walls (open arms). The entire maze sat on a stand $1 \mathrm{~m}$ above the floor. Each arm measured $30 \mathrm{~cm}$ long and $10 \mathrm{~cm}$ wide. Mice were allowed to explore the maze for 10 min while being recorded by digital video from above. The maze was wiped with $2-3 \%$ acetic acid, $70 \%$ ethanol and water between each test to eliminate odor trails. This test was always done following the open-field test. TopScan Lite software was used to measure the percent of time spent in open arms and speed. The time spent in open arm was used to evaluate anxiety-like behavior while speed and total arm entries were measured to evaluate overall locomotor activity ${ }^{14}$. The analysis was performed in 5 min intervals for the total 10 min exploration duration. Assessments of the digital recordings were done blind 
to the condition using TopScan Lite software. Statistical analysis was performed with unpaired ttest using GraphPad Prism 7 software. Data represent mean \pm standard error of the mean (SEM).

\section{Acknowledgements}

This work was supported by the National Institute of Child Health and Human Development and the National Institute of Mental Health (1U54 HD082008 to K.A.R. and I.M.E.), U.S. Army Medical Research and Materiel Command (W81XWH-15-1-0436 and W81XWH-15-1-0434 to I.M.E. and K.A.R.), and the National Institute of Health and the National Institute of Neurological Disorders and Stroke National Research Service Award Fellowship (1F31NS117178-01 to M.R). We thank Dr. Jonathan W. Lovelace for assistance with EEG analysis, and Stephen Brookshire for technical support; members of the Ethell, Binder, Razak, and Goel laboratories for helpful discussions; and Dr. David Carter for advice on confocal microscopy.

\section{Conflict of Interest}

The authors declare no competing financial interests.

\section{Figure Legends}

Figure 1. Astrocyte-specific deletion of FMRP during P14-P28 period.

a, Three transgenic mouse groups were used in this study: (1) ERT2-Cre ${ }^{G F A P}$ control (Ctrl WT) and ERT2-Cre ${ }^{G F A P} F m r 1^{\text {flox/y }}$ condition KO (cKO); (2) tdTomatoERT2-Cre ${ }^{G F A P}$ Ctrl WT and tdTomatoERT2-Cre ${ }^{G F A P} F m r 1^{\text {flox/y }} \mathrm{cKO}$; and (3) Pvalb-tdTomato-ERT2-Cre ${ }^{G F A P}$ Ctrl WT and Pvalb-tdvTomato-ERT2-Cre ${ }^{G F A P} F m r 1^{\text {flox/y }}$ cKO. To achieve FMRP deletion in astrocytes 
tamoxifen ( $0.5 \mathrm{mg})$ was intraperitoneally (IP) injected at P14 for 5 days; experiments were performed at P28 and P60 (adult) using mouse groups 1, 2 or 3. b, Analysis of Fmrl (exon 16/17) mRNA levels in the hippocampus (b.1.), cortex 1 (frontal cortical areas; b.2.), and cortex 2 (sensory cortical areas; b.3.) of Ctrl WT and cKO mice at P28 with qRT-PCR (Supplementary Figure 1a-c). Graphs show mean $\pm \operatorname{SEM}\left(\mathrm{n}=3-4\right.$ mice/group, ${ }^{*} \mathrm{p}<0.05 ;{ }^{*} \mathrm{p}<0.01$, t-test;

Supplementary Table 1). Fmrl mRNA levels were significantly reduced in all three brain areas of cKO mice compared to Ctrl WT. c-d, Confocal images showing tdTomato astrocytes (red) and FMRP immunoreactivity (green) in neurons (arrow) and astrocytes (arrowhead) of AuC of Ctrl WT (c) and cKO (d) mice at P28. e-g, Quantitative analysis of FMRP-positive (FMRP+) cell density (e), FMRP levels in FMRP+ cells (f) and FMRP levels in astrocytes (g) in the hippocampus, frontal cortex, and auditory cortex (Supplementary Figure 1d). Graphs show mean \pm SEM ( $\mathrm{n}=3$ mice/group, $* * * \mathrm{p}<0.001$, t-test; Supplementary Table 1$)$. While no significant differences were observed in FMRP+ cell density and FMRP intensity in FMRP+ cells between the genotypes, there was a significant decrease in FMRP levels in cKO astrocytes compared to Ctrl WT mice.

Figure 2. Developmental deletion of Fmrl from astrocytes affects PV levels and synaptic connectivity between PV and PC leading to reduced density of PV-expressing cells in the adult AuC of cKO mice.

a, qRT-PCR analysis of PV mRNA levels. Graph shows mean \pm SEM ( $n=3-4$ mice/group, ${ }^{*} \mathrm{p}<0.05 ;{ }^{*} \mathrm{p}<0.01$, t-test; Supplementary Table 2). PV mRNA levels are significantly decreased in the hippocampus and cortex of cKO mice compared to Ctrl WT. 
b, Western blots showing PV and beta-actin protein levels in lysates from hippocampus and cortex of Ctrl WT and cKO mice. Graph shows mean $\pm \operatorname{SEM}\left(\mathrm{n}=3-4 /\right.$ group, ${ }^{*} \mathrm{p}<0.05$;

***p<0.001, t-test; Supplementary Table 2). PV levels normalized to beta-actin levels are significantly reduced in the cortical samples of cKO mice compared to $\mathrm{Ctrl}$ WT c, Confocal images showing PV-positive pre-synaptic boutons (blue) immunolabelled with vGAT (red) and gephyrin (green) to visualize perisomatic inhibitory synaptic sites in L2-4 AuC of Ctrl WT and cKO mice. Scale bar, $20 \mu \mathrm{m}$. d-f, Quantitative analysis of vGAT/Gephyrin (d), PV/Gephyrin (e), and $\% \mathrm{PV} /$ Gephyrin synaptic puncta (f). Graphs show mean $\pm \operatorname{SEM}\left(\mathrm{n}=4\right.$ mice/group, ${ }^{*} \mathrm{p}<0.05$; **p $<0.01$, t-test; Supplementary Table 3). vGAT/Gephyrin, PV/Gephyrin, and \% PV/Gephyrin inhibitory synaptic sites are significantly reduced in AuC of cKO mice compared to Ctrl WT. g, Confocal images showing PV inhibitory interneurons expressing tdTomato (blue) immunolabelled with PSD95 (red) and vGlut1 (green) to visualize excitatory synaptic sites in L2-4 AuC of Ctrl WT and cKO mice. Scale bar, $20 \mu \mathrm{m} . \mathbf{h}$, Quantitative analysis of vGlut1/PSD95 puncta colocalization on tdTomato-expressing PV interneurons. Graphs show mean \pm SEM ( $n=2$ mice/group, ${ }^{*} \mathrm{p}<0.05$, t-test; Supplementary Table 3 ). Analysis shows that colocalization of vGlut1 and PSD95 on PV interneurons is significantly reduced in AuC of cKO mice compared to Ctrl WT. i, Schematic depiction of reduced excitatory innervation of PV cells (blue) by PC (green) and decreased PV-positive (blue) perisomatic innervation of PC (green) following Fmrl deletion in astrocytes (red). j, Confocal images showing PV immunoreactivity (red) and WFA+ PNN labeling (green) in AuC of adult Ctrl WT and cKO mice. k, Quantitative analysis of the density of $\mathrm{PV}, \mathrm{PNN}$, or $\mathrm{PV} / \mathrm{PNN}$ positive cells. Graphs show mean $\pm \mathrm{SEM}(\mathrm{n}=6$ mice/group, ${ }^{*} \mathrm{p}<0.05 ;{ }^{*} \mathrm{p}<0.01$, t-test; Supplementary Table 4). PV cell density was significantly reduced in L2/3 and L4 AuC of cKO mice compared to Ctrl WT. PNN cell density 
and PV/PNN co-localization were also significantly reduced in L4 AuC of cKO mice compared to Ctrl WT (note that only WFA+ cells were counted to measure PNN density).

\section{Figure 3. Characterization of baseline EEG power in the auditory and frontal cortex of adult cKO mice following developmental deletion of FMRP from astrocytes.}

Five min of baseline EEG data (in the absence of auditory stimulation) from electrodes implanted in AuC and FC of Ctrl WT $(n=10)$ and $\mathrm{cKO}(\mathrm{n}=9)$ mice (Supplementary Figure 1e) was recorded and FFT analysis was done to determine spectral power. a, Examples of $2 \mathrm{~s}$ segments of raw baseline EEG from AuC and FC of Ctrl WT and $\mathrm{CKO}$ mice. The enhanced high frequency oscillations can be visually observed in both $\mathrm{AuC}$ and $\mathrm{FC}$ of cKO mice. b, Power density $\left(\mu \mathrm{V}^{2} / \mathrm{Hz}\right)$ was calculated for each artifact-free segment using Fast Fourier Transform, followed by averaging of all segments for a given mouse. These individual averages then contributed to the grand average for each genotype ( $n=9-10$ per genotype). Significant differences between genotypes are observed in $\mathrm{AuC}$ and $\mathrm{FC}$ at gamma frequencies (low: 30-55 Hz, high: 65-100 Hz). Frequencies from 55-65 Hz were excluded in all analysis, as a $60 \mathrm{~Hz}$ notch filter was utilized to eliminate line noise. c, e, Average power in AuC (c) and FC (e) is expressed as the ratio of Ctrl WT levels. A value of 1 (horizontal black line) indicates no mean difference in power at that frequency between genotypes, whereas values $>1$ indicate $\mathrm{cKO}>\mathrm{Ctrl}$ $\mathrm{WT}$, and $<1$ indicates $\mathrm{cKO}<\mathrm{Ctrl} \mathrm{WT}$. The elevated gamma power (red) can be visualized in AuC and $\mathrm{FC}$ of cKO relative to Ctrl WT mice. d, f, Quantification of spectral power differences across genotypes. The values were divided into canonical frequency bands. MANCOVA analysis controlling for the effect of movement, revealed differences in the gamma range in $\mathrm{AuC}(\mathrm{d})$ and FC (f) of cKO mice after Bonferroni correction for multiple comparisons $\left({ }^{*} \mathrm{p}<0.05,{ }^{*} \mathrm{p}<0.01\right.$, 
$* * * \mathrm{p}<0.001, * * * * \mathrm{p}<0.0001$; Supplementary Table 5). The gamma band was further subdivided into low and high gamma revealing genotype differences in both bands. g, Power coupling of different oscillation frequencies. Graphs show Pearson's correlation (r) for AuC Delta/AuC Gamma (D1G1), AuC Theta/AuC Gamma (T1G1), and AuC Alpha/AuC Gamma (A1G1); FC Delta/FC Gamma (D2G2), FC Theta/FC Gamma (T2G2), and FC Alpha/FC Gamma (A2G2); AuC Delta/FC Gamma (D1G2), AuC Theta/FC Gamma (T1G2), and AuC Alpha/FC Gamma (A1G2); FC Delta/AuC Gamma (D2G1), FC Theta/AuC Gamma (T2G1), and FC Alpha/AuC Gamma (A2G1) power coupling $\left({ }^{*} \mathrm{p}<0.05 ;{ }^{*} \mathrm{p}<0.01\right.$, t-test; Supplementary Table 6$)$.

Figure 4. Developmental deletion of $F m r 1$ from astrocytes affects phase locking to time varying auditory stimuli (chirps), as well as non-phase locked single trial power (STP) in the auditory and frontal cortex of adult cKO mice.

The chirp stimulus (oscillogram shown at the bottom of this figure) is a $2 \mathrm{~s}$ broadband noise whose amplitude is modulated linearly by a frequency sweep with frequencies increasing from 1 to $100 \mathrm{~Hz}$. To reduce stimulus onset from overwhelming the early response, the chirp is preceded by a $1 \mathrm{~s}$ slow ramp of broadband noise. The ability of the cortical neural generators of EEG to follow this temporally dynamic stimulus is quantified by measuring the inter-trial phase coherence (ITPC, also known as phase locking factor). Trains of chirp stimuli were presented to each mouse 300 times. For each mouse, ITPC was measured to determine the degree of phase locking across trials. a-b, Grand average matrices were calculated for each genotype (left and middle panels), and then Ctrl WT ( $\mathrm{n}=10)$ ITPC values were subtracted from $\mathrm{cKO}(\mathrm{n}=9)$ values (right panels) for $\mathrm{AuC} \mathrm{(a)} \mathrm{and} \mathrm{FC}(\mathrm{b})$. Blue areas indicating $\mathrm{cKO}<\mathrm{Ctrl} \mathrm{WT}$, green areas no difference, and red $\mathrm{cKO}>\mathrm{Ctrl}$ WT. Statistical cluster analysis reveals contiguous time $\mathrm{x}$ 
frequency regions that are significantly different between genotypes. Black solid contours (mean negative difference) and black dashed contours (mean positive difference) indicate clusters with significant differences. cKO mice express statistically significant decrease in ITPC at beta (13-30 $\mathrm{Hz}$ ) and gamma frequencies $(30-100 \mathrm{~Hz}$, blue). c-d, For each mouse, single-trial power (STP) was measured to determine the average total non-phase locked power during chirp train presentation. Grand average matrices were calculated for each genotype (Supplementary Figure 2), and then Ctrl WT (n=10) STP values were subtracted from $\mathrm{cKO}(\mathrm{n}=9)$ values for $\mathrm{AuC}(\mathrm{c})$ and FC (d). Statistical cluster analysis reveals contiguous time $\mathrm{x}$ frequency regions that are significantly different between genotypes. Black dashed contour indicates these significant clusters. Consistent with the increase in gamma power changes in baseline EEGs, cKO mice express statistically significant increase in non-phase gamma power range (30-100 Hz, red) throughout sound presentation in both $\mathrm{AuC}$ and $\mathrm{FC}$.

\section{Figure 5. Developmental deletion of Fmr1 from astrocytes affects induced (baseline corrected) power in response to sound presentation in the auditory and frontal cortex of adult cKO mice.}

$\mathbf{a}-\mathbf{b}$, Example traces of responses in $\mathrm{AuC}$ and $\mathrm{FC}$ to single broadband noise presentation repeated at $0.25 \mathrm{~Hz}$ (a) and sound train presented at $4 \mathrm{~Hz}$ (b) for Ctrl WT and cKO mice. Red arrow indicates onset of $100 \mathrm{~ms}$ broadband noise. c-d, Grand average difference plot of baseline corrected sound-induced power during single sound presentation at $0.25 \mathrm{~Hz}$ and sound presentations in $4 \mathrm{~Hz}$ train were calculated for each genotype (Supplementary Figure 3), and differences between genotypes (cKO - Ctrl WT) are presented here. In both AuC and FC, increased induced power is observed in the $\mathrm{cKO}$ mice in response to the sound as well as after 
sound presentation, indicating increased onset and on-going activity. In addition, increased onset and on-going activity is also observed in $\mathrm{AuC}$ and $\mathrm{FC}$ of the $\mathrm{cKO}$ mice at $\sim 10-40 \mathrm{~Hz}$ during all four sounds in the $4 \mathrm{~Hz}$ train.

Figure 6. Postnatal deletion of Fmr1 from astrocytes alters synaptic GABAA receptor levels in the developing hippocampus and cortex.

a, Heatmap of genes from the Glial Profiling NanoString Panel (Supplementary Figure 4) encoding GABAergic proteins in the hippocampus of $\mathrm{cKO}$ and $\mathrm{Ctrl}$ WT mice. Heatmap shows normalized gene expression data from low expression (yellow) to high expression (blue) and is generated using nSolver software. b-d, Quantitative analysis of mRNA levels of GABAergic genes. Analysis of mRNA expression in cortical samples (Supplementary Figure 5) was performed with qRT-PCR. Graphs show mean \pm SEM $\left(\mathrm{n}=4\right.$ mice/group; ${ }^{*} \mathrm{p}<0.05 ; * * \mathrm{p}<0.01, \mathrm{t}-$ test; Supplementary Table 7). No significant differences were observed in mRNA levels of Gad1 and Gad2 (b); Gabra4 (d.1.), Gabra5 (d.2.), and Gabrd (d.3.) in the hippocampus (NanoString) and cortex (qRT-PCR) of cKO mice compared to Ctrl WT. However, there is a significant decrease in synaptic $\mathrm{GABA}_{\mathrm{A}}$ receptor subunits: Gabra1 (c.1.), Gabra3 (c.2.), and Gabrg2 (c.3.) in the hippocampus (NanoString) and cortex (qRT-PCR) of cKO mice compared to Ctrl WT. e-f, Western blots showing $\mathrm{GABA}_{\mathrm{A}} \mathrm{R} \gamma 2$ and $\mathrm{GABA}_{\mathrm{A}} \mathrm{R} \alpha 5$ protein levels in lysates from hippocampus and cortex of Ctrl WT and cKO mice. Graphs show mean $\pm \operatorname{SEM}\left(n=4 /\right.$ group, ${ }^{*} \mathrm{p}<0.05$; ${ }^{* *} \mathrm{p}<0.01 ; * * * \mathrm{p}<0.001, \mathrm{t}$-test; Supplementary Table 7). Levels of synaptic GABA $\mathrm{R} \gamma 2$ but not extrasynaptic $\mathrm{GABA}_{\mathrm{A}} \mathrm{R} \alpha 5$ subunit were significantly reduced in hippocampus and cortex of $\mathrm{cKO}$ mice compared to Ctrl WT. 


\section{Figure 7. Astrocytic GABA levels and expression of GABA-synthesizing enzymes are} significantly upregulated following the developmental deletion of FMRP in astrocytes.

a, Confocal images showing Glutamine Synthetase (GS, red) and GABA (green) immunoreactivity in superficial layers of $\mathrm{AuC}$ of $\mathrm{Ctrl}$ WT and $\mathrm{cKO}$ mice. Arrows indicate GSlabeled cell bodies of astrocytes. b, Quantitative analysis of the intensity of GABA immunoreactivity in GS-labeled astrocytes. Graphs show mean $\pm \operatorname{SEM}(n=4$ mice/group, $* * \mathrm{p}<0.01$, t-test; Supplementary Table 8). GABA levels in GS-labeled astrocytes were significantly increased in the hippocampus and cortex of cKO mice compared to Ctrl WT. c-d, Immunoblot for GAD65/67, Aldha1 and beta-actin (c) and GABA immunodetection (d) in cultured astrocytes from WT and Fmr1 KO mice. Graphs show mean \pm SEM ( $n=5-6$ mice/group, ${ }^{*} \mathrm{p}<0.05$, t-test; Supplementary Table 8 ). e, qRT-PCR analysis showed an increase in GFAP mRNA levels in the cortex of cKO mice compared to Ctrl WT. Graphs show mean \pm SEM ( $\mathrm{n}=4$ mice/group, ${ }^{* *} \mathrm{p}<0.01$, $\mathrm{t}$-test; Supplementary Table 8$) . \mathbf{f}$, Western blots showing GFAP protein levels in lysates from hippocampus and cortex of Ctrl WT and cKO mice. No significant differences were observed in GFAP protein levels in the hippocampus. However, there is a trend for increase in cortical sample 1 and a significant increase in GFAP protein levels in cortical sample 2 of cKO mice compared to Ctrl WT. Graphs show mean $\pm \operatorname{SEM}(n=4$ mice/group, ${ }^{*}{ }^{*} \mathrm{p}<0.01$, t-test; Supplementary Table 8 ).

\section{Figure 8. Developmental deletion of Fmr1 in astrocytes leads to increased locomotor} activity and decreased socialization in adult cKO mice.

$\mathbf{a - b}$, Graphs demonstrate the performance of mice in the elevated plus maze. Locomotor activity is measured by total arm entries and speed (a). Anxiety-like behavior is measured by percentage 
of time in open arms and time spent in open arm per entry (b). Graphs show mean \pm SEM ( $n=8$ mice/group, ${ }^{* *} \mathrm{p}<0.01 ; * * * \mathrm{p}<0.001$, t-test; Supplementary Table 9). c-d, Graphs demonstrate the performance of mice in the open field. Locomotor activity is measured by total line crosses and speed (c). Anxiety-like behavior is measured by percentage of time in thigmotaxis and time spent in center per entry (d). Graphs show mean $\pm \operatorname{SEM}\left(\mathrm{n}=8\right.$ mice/group, ${ }^{* *} \mathrm{p}<0.01 ; * * * \mathrm{p}<0.001, \mathrm{t}$ test; Supplementary Table 9). cKO exhibit higher locomotor activity than Ctrl WT mice (a, c). No changes in anxiety-like behaviors are observed (b, d). e-f, Graphs demonstrate the sociability (e) and social novelty preference (f) of mice in the social novelty test. Session 1: preference between an empty chamber and a chamber with S1 mouse during sociability test. Session 2: preference between a chamber with a novel mouse S2 and a chamber with a familiar mouse S1 during social novelty test. Left panels, graphs show time spent in the chambers (mean $\pm \mathrm{SEM}$, $\mathrm{n}=8 \mathrm{mice} /$ group, ${ }^{*} \mathrm{p}<0.05 ;{ }^{*} \mathrm{p}<0.01,{ }^{*} * \mathrm{p}<0.001$, two-way ANOVA, Bonferroni post hoc test; Supplementary Table 10). Right panels, graphs show sociability index (e) or social novelty preference index (f) $(* * \mathrm{p}<0.01, * * * * \mathrm{p}<0.001$, t-test; Supplementary Table 10). In Session 1, Ctrl WT mice prefer spending time with S1 mouse compared with time in the empty chamber. cKO mice show impaired sociability, reduced sociability index and spend significantly more time in the empty chamber than with S1 mouse. In Session 2, Ctrl WT mice spend significantly more time with novel S2 mouse than with familiar S1 mouse, indicating normal social novelty. cKO mice spend the same amount of time in S1 and S2 chambers, and show reduced social novelty index, indicating impaired social novelty preference. $\mathbf{g}$, Schematic depiction of phenotypes observed following deletion of Fmrl from astrocytes during P14-P28 postnatal developmental period. cKO mice exhibit reduced excitatory innervation of PV cell (blue) by PC (green) and decreased PV-positive (blue) perisomatic innervation of PC (green) and GABA $\mathrm{R}$ levels at P28 
following Fmrl deletion in astrocytes (red). Adult cKO mice also show reduced chirp-evoked gamma synchronization, enhanced on-set and on-going gamma power, impaired socialization and increased locomotor activity.

\section{References}

1. Crawford, D.C., Acuna, J.M. \& Sherman, S.L. FMR1 and the fragile X syndrome: human genome epidemiology review. Genet Med 3, 359-371 (2001).

2. Sutcliffe, J.S. et al. DNA methylation represses FMR-1 transcription in fragile X syndrome. Hum Mol Genet 1, 397-400 (1992).

3. Verkerk, A.J. et al. Identification of a gene (FMR-1) containing a CGG repeat coincident with a breakpoint cluster region exhibiting length variation in fragile $\mathrm{X}$ syndrome. Cell 65, 905-914 (1991).

4. Penagarikano, O., Mulle, J.G. \& Warren, S.T. The pathophysiology of fragile x syndrome. Annu Rev Genomics Hum Genet 8, 109-129 (2007).

5. Rais, M., Binder, D.K., Razak, K.A. \& Ethell, I.M. Sensory Processing Phenotypes in Fragile X Syndrome. ASN Neuro 10, 1759091418801092 (2018).

6. Castren, M., Paakkonen, A., Tarkka, I.M., Ryynanen, M. \& Partanen, J. Augmentation of auditory N1 in children with fragile X syndrome. Brain Topogr 15, 165-171 (2003).

7. Ethridge, L.E. et al. Reduced habituation of auditory evoked potentials indicate cortical hyper-excitability in Fragile X Syndrome. Transl Psychiatry 6, e787 (2016).

8. Schneider, A. et al. Electrocortical changes associated with minocycline treatment in fragile X syndrome. J Psychopharmacol 27, 956-963 (2013). 
9. Antoine, M.W., Langberg, T., Schnepel, P. \& Feldman, D.E. Increased ExcitationInhibition Ratio Stabilizes Synapse and Circuit Excitability in Four Autism Mouse Models. Neuron 101, 648-661 e644 (2019).

10. Goncalves, J.T., Anstey, J.E., Golshani, P. \& Portera-Cailliau, C. Circuit level defects in the developing neocortex of Fragile X mice. Nat Neurosci 16, 903-909 (2013).

11. Zhang, Y. et al. Dendritic channelopathies contribute to neocortical and sensory hyperexcitability in Fmr1(-/y) mice. Nat Neurosci 17, 1701-1709 (2014).

12. Gibson, J.R., Bartley, A.F., Hays, S.A. \& Huber, K.M. Imbalance of neocortical excitation and inhibition and altered UP states reflect network hyperexcitability in the mouse model of fragile X syndrome. J Neurophysiol 100, 2615-2626 (2008).

13. Goel, A. et al. Impaired perceptual learning in a mouse model of Fragile X syndrome is mediated by parvalbumin neuron dysfunction and is reversible. Nat Neurosci 21, 14041411 (2018).

14. Lovelace, J.W. et al. Deletion of Fmr1 from Forebrain Excitatory Neurons Triggers Abnormal Cellular, EEG, and Behavioral Phenotypes in the Auditory Cortex of a Mouse Model of Fragile X Syndrome. Cereb Cortex 30, 969-988 (2020).

15. Wen, T.H. et al. Genetic Reduction of Matrix Metalloproteinase-9 Promotes Formation of Perineuronal Nets Around Parvalbumin-Expressing Interneurons and Normalizes Auditory Cortex Responses in Developing Fmr1 Knock-Out Mice. Cereb Cortex 28, 3951-3964 (2018).

16. Adusei, D.C., Pacey, L.K., Chen, D. \& Hampson, D.R. Early developmental alterations in GABAergic protein expression in fragile X knockout mice. Neuropharmacology 59, 167-171 (2010). 
17. Braat, S. \& Kooy, R.F. The GABAA Receptor as a Therapeutic Target for Neurodevelopmental Disorders. Neuron 86, 1119-1130 (2015).

18. Curia, G., Papouin, T., Seguela, P. \& Avoli, M. Downregulation of tonic GABAergic inhibition in a mouse model of fragile X syndrome. Cereb Cortex 19, 1515-1520 (2009).

19. D'Hulst, C. et al. Decreased expression of the GABAA receptor in fragile X syndrome. Brain Res 1121, 238-245 (2006).

20. Hays, S.A., Huber, K.M. \& Gibson, J.R. Altered neocortical rhythmic activity states in Fmr1 KO mice are due to enhanced mGluR5 signaling and involve changes in excitatory circuitry. J Neurosci 31, 14223-14234 (2011).

21. Contractor, A., Klyachko, V.A. \& Portera-Cailliau, C. Altered Neuronal and Circuit Excitability in Fragile X Syndrome. Neuron 87, 699-715 (2015).

22. Darnell, J.C. et al. FMRP stalls ribosomal translocation on mRNAs linked to synaptic function and autism. Cell 146, 247-261 (2011).

23. Edbauer, D. et al. Regulation of synaptic structure and function by FMRP-associated microRNAs miR-125b and miR-132. Neuron 65, 373-384 (2010).

24. Zhang, Y.Q. et al. Drosophila fragile X-related gene regulates the MAP1B homolog Futsch to control synaptic structure and function. Cell 107, 591-603 (2001).

25. Ishibashi, M., Egawa, K. \& Fukuda, A. Diverse Actions of Astrocytes in GABAergic Signaling. Int J Mol Sci 20 (2019).

26. Ullian, E.M., Christopherson, K.S. \& Barres, B.A. Role for glia in synaptogenesis. Glia 47, 209-216 (2004).

27. Tan, C.X., Burrus Lane, C.J. \& Eroglu, C. Role of astrocytes in synapse formation and maturation. Curr Top Dev Biol 142, 371-407 (2021). 
28. Higashimori, H. et al. Selective Deletion of Astroglial FMRP Dysregulates Glutamate Transporter GLT1 and Contributes to Fragile X Syndrome Phenotypes In Vivo. $J$ Neurosci 36, 7079-7094 (2016).

29. Jacobs, S. \& Doering, L.C. Astrocytes prevent abnormal neuronal development in the fragile x mouse. J Neurosci 30, 4508-4514 (2010).

30. Pacey, L.K. \& Doering, L.C. Developmental expression of FMRP in the astrocyte lineage: implications for fragile X syndrome. Glia 55, 1601-1609 (2007).

31. Jin, S.X. et al. Astroglial FMRP modulates synaptic signaling and behavior phenotypes in FXS mouse model. Glia 69, 594-608 (2021).

32. Dorrn, A.L., Yuan, K., Barker, A.J., Schreiner, C.E. \& Froemke, R.C. Developmental sensory experience balances cortical excitation and inhibition. Nature 465, 932-936 (2010).

33. Oswald, A.M. \& Reyes, A.D. Maturation of intrinsic and synaptic properties of layer 2/3 pyramidal neurons in mouse auditory cortex. J Neurophysiol 99, 2998-3008 (2008).

34. Oswald, A.M. \& Reyes, A.D. Development of inhibitory timescales in auditory cortex. Cereb Cortex 21, 1351-1361 (2011).

35. Sun, Y.J. et al. Fine-tuning of pre-balanced excitation and inhibition during auditory cortical development. Nature 465, 927-931 (2010).

36. Razak, K.A., Binder, D.K. \& Ethell, I.M. Neural Correlates of Auditory Hypersensitivity in Fragile X Syndrome. Front Psychiatry 12, 720752 (2021).

37. Lovelace, J.W., Ethell, I.M., Binder, D.K. \& Razak, K.A. Translation-relevant EEG phenotypes in a mouse model of Fragile X Syndrome. Neurobiol Dis 115, 39-48 (2018). 
38. Contractor, A., Ethell, I.M. \& Portera-Cailliau, C. Cortical interneurons in autism. Nat Neurosci 24, 1648-1659 (2021).

39. Filice, F., Janickova, L., Henzi, T., Bilella, A. \& Schwaller, B. The Parvalbumin Hypothesis of Autism Spectrum Disorder. Front Cell Neurosci 14, 577525 (2020).

40. Hashemi, E., Ariza, J., Rogers, H., Noctor, S.C. \& Martinez-Cerdeno, V. The Number of Parvalbumin-Expressing Interneurons Is Decreased in the Prefrontal Cortex in Autism. Cereb Cortex 27, 1931-1943 (2017).

41. Lunden, J.W., Durens, M., Phillips, A.W. \& Nestor, M.W. Cortical interneuron function in autism spectrum condition. Pediatr Res 85, 146-154 (2019).

42. Marin, O. Interneuron dysfunction in psychiatric disorders. Nat Rev Neurosci 13, 107$120(2012)$.

43. Rossignol, E. Genetics and function of neocortical GABAergic interneurons in neurodevelopmental disorders. Neural Plast 2011, 649325 (2011).

44. Buzsaki, G. \& Draguhn, A. Neuronal oscillations in cortical networks. Science 304, 1926-1929 (2004).

45. Sinclair, D., Oranje, B., Razak, K.A., Siegel, S.J. \& Schmid, S. Sensory processing in autism spectrum disorders and Fragile $\mathrm{X}$ syndrome-From the clinic to animal models. Neurosci Biobehav Rev 76, 235-253 (2017).

46. Wang, J. et al. Resting state EEG abnormalities in autism spectrum disorders. J Neurodev Disord 5, 24 (2013).

47. Ethridge, L.E. et al. Neural synchronization deficits linked to cortical hyper-excitability and auditory hypersensitivity in fragile X syndrome. Mol Autism 8, 22 (2017). 
48. Lovelace, J.W. et al. Matrix metalloproteinase-9 deletion rescues auditory evoked potential habituation deficit in a mouse model of Fragile X Syndrome. Neurobiol Dis 89, 126-135 (2016).

49. Rotschafer, S. \& Razak, K. Altered auditory processing in a mouse model of fragile X syndrome. Brain Res 1506, 12-24 (2013).

50. Gao, F. et al. Impaired GABA Neural Circuits Are Critical for Fragile X Syndrome. Neural Plast 2018, 8423420 (2018).

51. Gatto, C.L., Pereira, D. \& Broadie, K. GABAergic circuit dysfunction in the Drosophila Fragile X syndrome model. Neurobiol Dis 65, 142-159 (2014).

52. Sabanov, V. et al. Impaired GABAergic inhibition in the hippocampus of Fmr1 knockout mice. Neuropharmacology 116, 71-81 (2017).

53. Lin, R.C., Polsky, K. \& Matesic, D.F. Expression of gamma-aminobutyric acid immunoreactivity in reactive astrocytes after ischemia-induced injury in the adult forebrain. Brain Res 600, 1-8 (1993).

54. Lee, E., Lee, J. \& Kim, E. Excitation/Inhibition Imbalance in Animal Models of Autism Spectrum Disorders. Biol Psychiatry 81, 838-847 (2017).

55. Selby, L., Zhang, C. \& Sun, Q.Q. Major defects in neocortical GABAergic inhibitory circuits in mice lacking the fragile X mental retardation protein. Neurosci Lett 412, 227 232 (2007).

56. Favuzzi, E. et al. GABA-receptive microglia selectively sculpt developing inhibitory circuits. Cell 184, $5686(2021)$.

57. Rais, M. et al. Functional consequences of postnatal interventions in a mouse model of Fragile X syndrome. Neurobiol Dis, 105577 (2021). 
58. Cardin, J.A. et al. Driving fast-spiking cells induces gamma rhythm and controls sensory responses. Nature 459, 663-667 (2009).

59. Chen, G. et al. Distinct Inhibitory Circuits Orchestrate Cortical beta and gamma Band Oscillations. Neuron 96, 1403-1418 e1406 (2017).

60. Guyon, N. et al. Network Asynchrony Underlying Increased Broadband Gamma Power. J Neurosci 41, 2944-2963 (2021).

61. Sohal, V.S., Zhang, F., Yizhar, O. \& Deisseroth, K. Parvalbumin neurons and gamma rhythms enhance cortical circuit performance. Nature 459, 698-702 (2009).

62. Volman, V., Behrens, M.M. \& Sejnowski, T.J. Downregulation of parvalbumin at cortical GABA synapses reduces network gamma oscillatory activity. J Neurosci 31, 18137-18148 (2011).

63. Brosch, M., Budinger, E. \& Scheich, H. Stimulus-related gamma oscillations in primate auditory cortex. J Neurophysiol 87, 2715-2725 (2002).

64. Cervenka, M.C., Nagle, S. \& Boatman-Reich, D. Cortical high-gamma responses in auditory processing. Am J Audiol 20, 171-180 (2011).

65. Franowicz, M.N. \& Barth, D.S. Comparison of evoked potentials and high-frequency (gamma-band) oscillating potentials in rat auditory cortex. J Neurophysiol 74, 96-112 (1995).

66. Jeschke, M., Lenz, D., Budinger, E., Herrmann, C.S. \& Ohl, F.W. Gamma oscillations in gerbil auditory cortex during a target-discrimination task reflect matches with short-term memory. Brain Res 1220, 70-80 (2008). 
67. Desmedt, J.E. \& Tomberg, C. Transient phase-locking of $40 \mathrm{~Hz}$ electrical oscillations in prefrontal and parietal human cortex reflects the process of conscious somatic perception. Neurosci Lett 168, 126-129 (1994).

68. Jokeit, H. \& Makeig, S. Different event-related patterns of gamma-band power in brain waves of fast- and slow-reacting subjects. Proc Natl Acad Sci U S A 91, 6339-6343 (1994).

69. Tallon-Baudry, C., Bertrand, O., Delpuech, C. \& Pernier, J. Stimulus specificity of phaselocked and non-phase-locked $40 \mathrm{~Hz}$ visual responses in human. J Neurosci 16, 42404249 (1996).

70. Metherate, R. \& Cruikshank, S.J. Thalamocortical inputs trigger a propagating envelope of gamma-band activity in auditory cortex in vitro. Exp Brain Res 126, 160-174 (1999).

71. Oliveira, J.F., Sardinha, V.M., Guerra-Gomes, S., Araque, A. \& Sousa, N. Do stars govern our actions? Astrocyte involvement in rodent behavior. Trends Neurosci 38, 535$549(2015)$

72. Clarke, L.E. \& Barres, B.A. Emerging roles of astrocytes in neural circuit development. Nat Rev Neurosci 14, 311-321 (2013).

73. Nguyen, A.Q. et al. Astrocytic Ephrin-B1 Controls Excitatory-Inhibitory Balance in Developing Hippocampus. J Neurosci 40, 6854-6871 (2020).

74. Wallingford, J., Scott, A.L., Rodrigues, K. \& Doering, L.C. Altered Developmental Expression of the Astrocyte-Secreted Factors Hevin and SPARC in the Fragile X Mouse Model. Front Mol Neurosci 10, 268 (2017). 
75. Higashimori, H. et al. Astroglial FMRP-dependent translational down-regulation of mGluR5 underlies glutamate transporter GLT1 dysregulation in the fragile X mouse. Hum Mol Genet 22, 2041-2054 (2013).

76. Olmos-Serrano, J.L. et al. Defective GABAergic neurotransmission and pharmacological rescue of neuronal hyperexcitability in the amygdala in a mouse model of fragile $\mathrm{X}$ syndrome. J Neurosci 30, 9929-9938 (2010).

77. Zhang, N. et al. Decreased surface expression of the delta subunit of the GABAA receptor contributes to reduced tonic inhibition in dentate granule cells in a mouse model of fragile X syndrome. Exp Neurol 297, 168-178 (2017).

78. D'Hulst, C. et al. Expression of the GABAergic system in animal models for fragile X syndrome and fragile X associated tremor/ataxia syndrome (FXTAS). Brain Res 1253, 176-183 (2009).

79. Gantois, I. et al. Expression profiling suggests underexpression of the GABA(A) receptor subunit delta in the fragile X knockout mouse model. Neurobiol Dis 21, 346-357 (2006).

80. D'Hulst, C. et al. Positron Emission Tomography (PET) Quantification of GABAA Receptors in the Brain of Fragile X Patients. PLoS One 10, e0131486 (2015).

81. Erickson, C.A. et al. An open-label naturalistic pilot study of acamprosate in youth with autistic disorder. J Child Adolesc Psychopharmacol 21, 565-569 (2011).

82. Telias, M., Segal, M. \& Ben-Yosef, D. Immature Responses to GABA in Fragile X Neurons Derived from Human Embryonic Stem Cells. Front Cell Neurosci 10, 121 (2016).

83. Kwak, H. et al. Astrocytes Control Sensory Acuity via Tonic Inhibition in the Thalamus. Neuron 108, 691-706 e610 (2020). 
84. Yoon, B.E. \& Lee, C.J. GABA as a rising gliotransmitter. Front Neural Circuits 8, 141 (2014).

85. Semyanov, A., Walker, M.C. \& Kullmann, D.M. GABA uptake regulates cortical excitability via cell type-specific tonic inhibition. Nat Neurosci 6, 484-490 (2003).

86. Men, Y. et al. Astroglial FMRP deficiency cell-autonomously up-regulates miR-128 and disrupts developmental astroglial mGluR5 signaling. Proc Natl Acad Sci U S A 117, 25092-25103 (2020).

87. Mientjes, E.J. et al. The generation of a conditional Fmr1 knock out mouse model to study Fmrp function in vivo. Neurobiol Dis 21, 549-555 (2006).

88. Nikolakopoulou, A.M. et al. Astrocytic Ephrin-B1 Regulates Synapse Remodeling Following Traumatic Brain Injury. ASN Neuro 8, 1-18 (2016).

89. Schildge, S., Bohrer, C., Beck, K. \& Schachtrup, C. Isolation and culture of mouse cortical astrocytes. J Vis $\operatorname{Exp}(2013)$.

90. Paxinos, G. \& Franklin, K.B.J. The mouse brain in stereotaxic coordinates, Edn. Compact 2nd. (Elsevier Academic Press, Amsterdam ; Boston; 2004).

91. Koeppen, J. et al. Functional Consequences of Synapse Remodeling Following Astrocyte-Specific Regulation of Ephrin-B1 in the Adult Hippocampus. J Neurosci 38, 5710-5726 (2018).

92. Anderson, L.A., Christianson, G.B. \& Linden, J.F. Mouse auditory cortex differs from visual and somatosensory cortices in the laminar distribution of cytochrome oxidase and acetylcholinesterase. Brain Res 1252, 130-142 (2009).

93. Bergersen, K.V., Barnes, A., Worth, D., David, C. \& Wilson, E.H. Targeted Transcriptomic Analysis of C57BL/6 and BALB/c Mice During Progressive Chronic 
Toxoplasma gondii Infection Reveals Changes in Host and Parasite Gene Expression Relating to Neuropathology and Resolution. Front Cell Infect Microbiol 11, 645778 (2021).

94. Danaher, P. et al. Gene expression markers of Tumor Infiltrating Leukocytes. $J$ Immunother Cancer 5, 18 (2017).

95. Artieda, J. et al. Potentials evoked by chirp-modulated tones: a new technique to evaluate oscillatory activity in the auditory pathway. Clin Neurophysiol 115, 699-709 (2004).

96. Perez-Alcazar, M. et al. Chirp-evoked potentials in the awake and anesthetized rat. A procedure to assess changes in cortical oscillatory activity. Exp Neurol 210, 144-153 (2008).

97. Purcell, D.W., John, S.M., Schneider, B.A. \& Picton, T.W. Human temporal auditory acuity as assessed by envelope following responses. J Acoust Soc Am 116, 3581-3593 (2004).

98. Maris, E. \& Oostenveld, R. Nonparametric statistical testing of EEG- and MEG-data. $J$ Neurosci Methods 164, 177-190 (2007).

99. Fu, Y., Kaneko, M., Tang, Y., Alvarez-Buylla, A. \& Stryker, M.P. A cortical disinhibitory circuit for enhancing adult plasticity. Elife 4, e05558 (2015).

100. Niell, C.M. \& Stryker, M.P. Modulation of visual responses by behavioral state in mouse visual cortex. Neuron 65, 472-479 (2010).

101. Nygaard, K.R., Maloney, S.E. \& Dougherty, J.D. Erroneous inference based on a lack of preference within one group: Autism, mice, and the social approach task. Autism Res 12, $1171-1183$ (2019). 
bioRxiv preprint doi: https://doi.org/10.1101/2022.02.08.479618; this version posted February 9, 2022. The copyright holder for this preprint (which was not certified by peer review) is the author/funder. All rights reserved. No reuse allowed without permission.

102. Spongr, V.P., Flood, D.G., Frisina, R.D. and Salvi, R.J., Quantitative measures of hair cell loss in CBA and C57BL/6 mice throughout their life spans. The Journal of the Acoustical Society of America, 101(6), 3546-3553 (1997). 


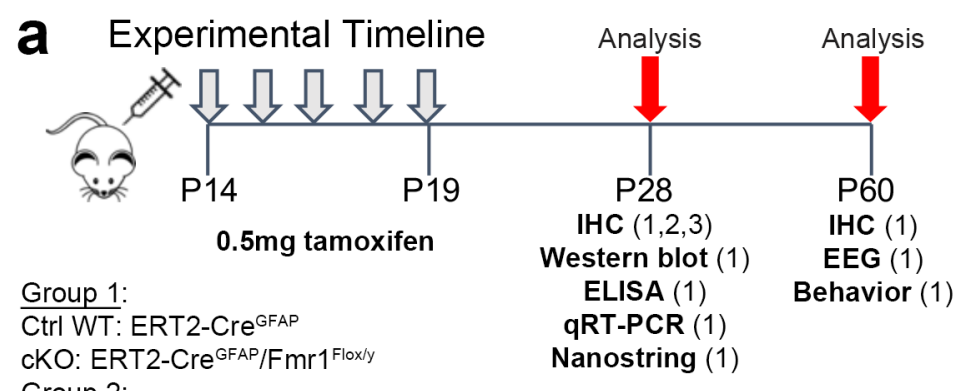

Group 2:

Ctrl WT: tdTomato-ERT2-Cre GFAP

cKO: tdTomato-ERT2-Cre ${ }^{\text {GFAP/Fmr1Flox/y }}$

Group 3:

Ctrl WT: Pvalb-tdTomato-ERT2-Cre GFAP

cKO: Pvalb-tdTomato-ERT2-Cre ${ }^{\text {GFAP/Fmr1Flox/y }}$

b $\quad F m r 1$ (exon 16/17) mRNA levels
B.1. Hippocampus
B.2. Cortex 1
B.3. Cortex 2
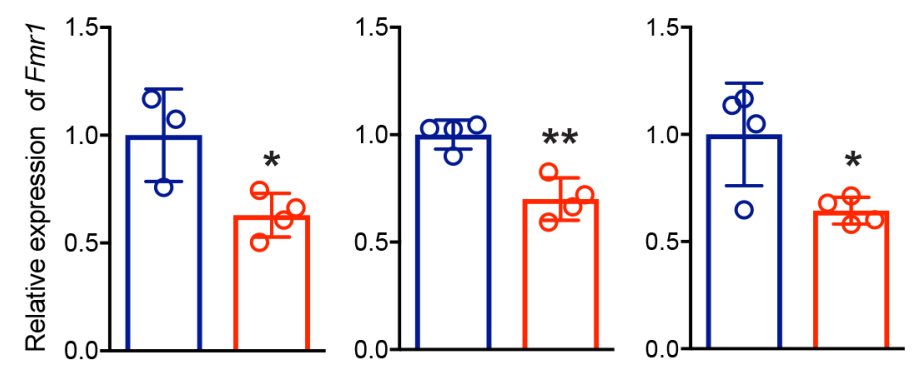

C Ctrl WT (tdTomato-ERT2-Cre ${ }^{\text {GFAP) }}$

P28 Cortex
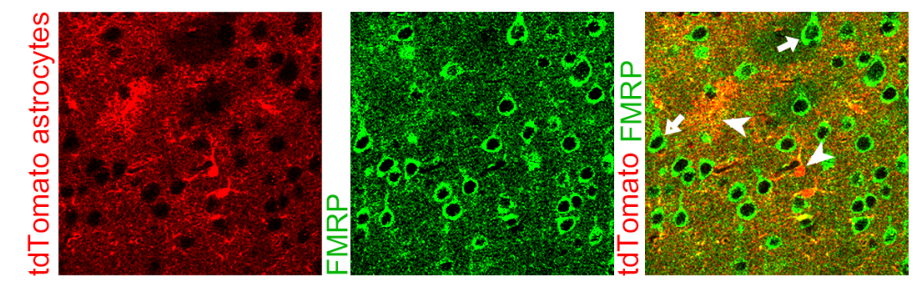

d cKO (tdTomato-ERT2-Cre ${ }^{\text {GFAP }} /$ Fmr1 ${ }^{\text {Flox/y }}$ )
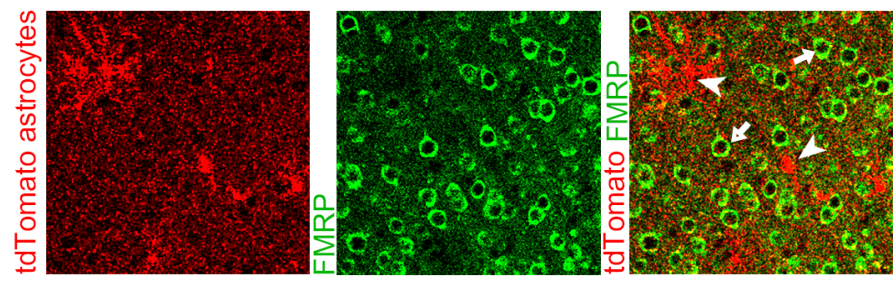
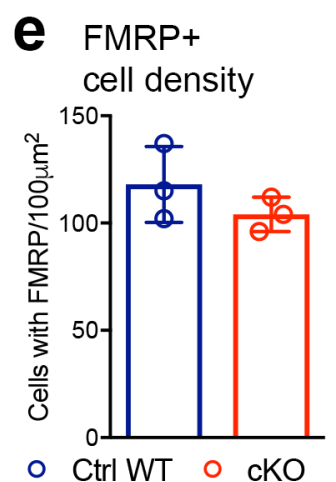

f FMRP levels in $\mathbf{g}$ FMRP+ cells

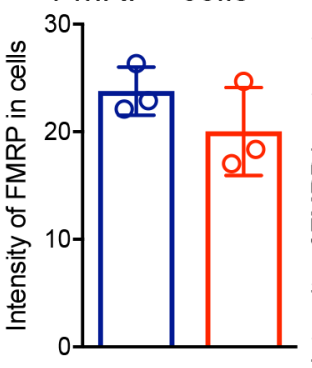

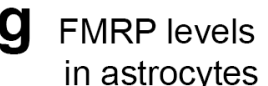

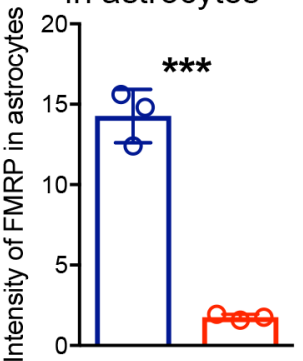


bioRxiy preprint doi: https://doi.org/10.1101/2022.02.08.479618; this version potted February 9, 2022. The copyright holder for this preprint

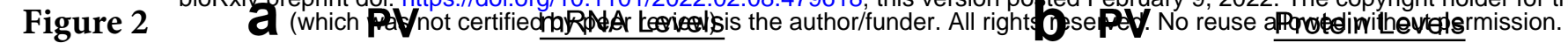

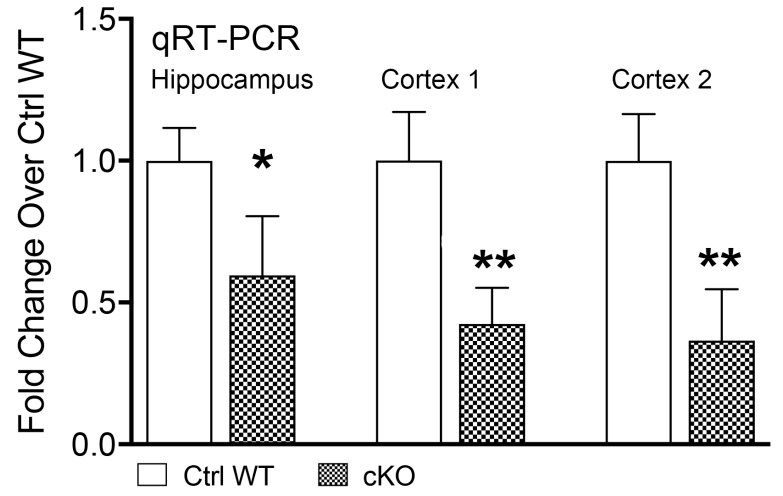

C Inhibitory perisomatic synapses

PV vGAT Gephyrin perisomatic puncta P28 Au Cortex
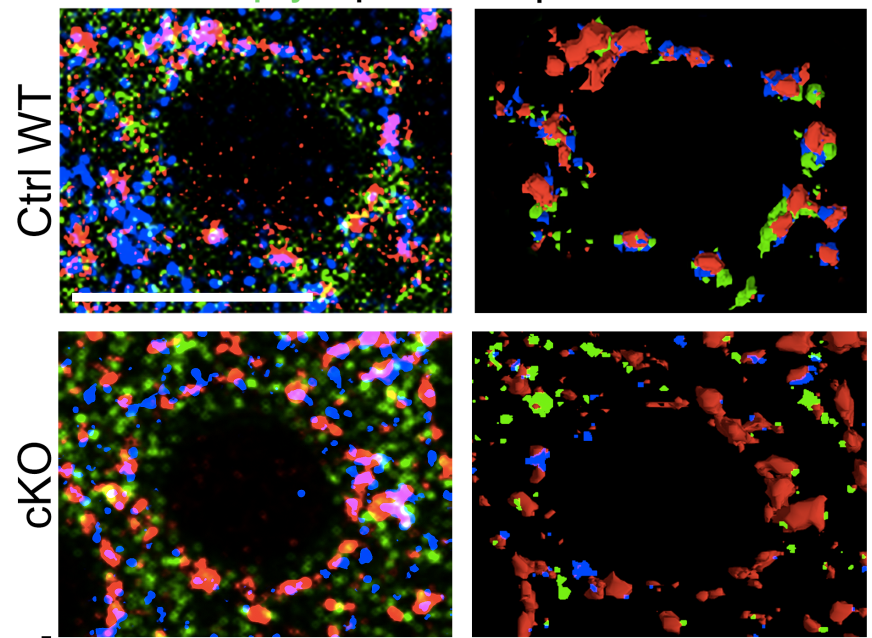

d vGAT/Gephyrin
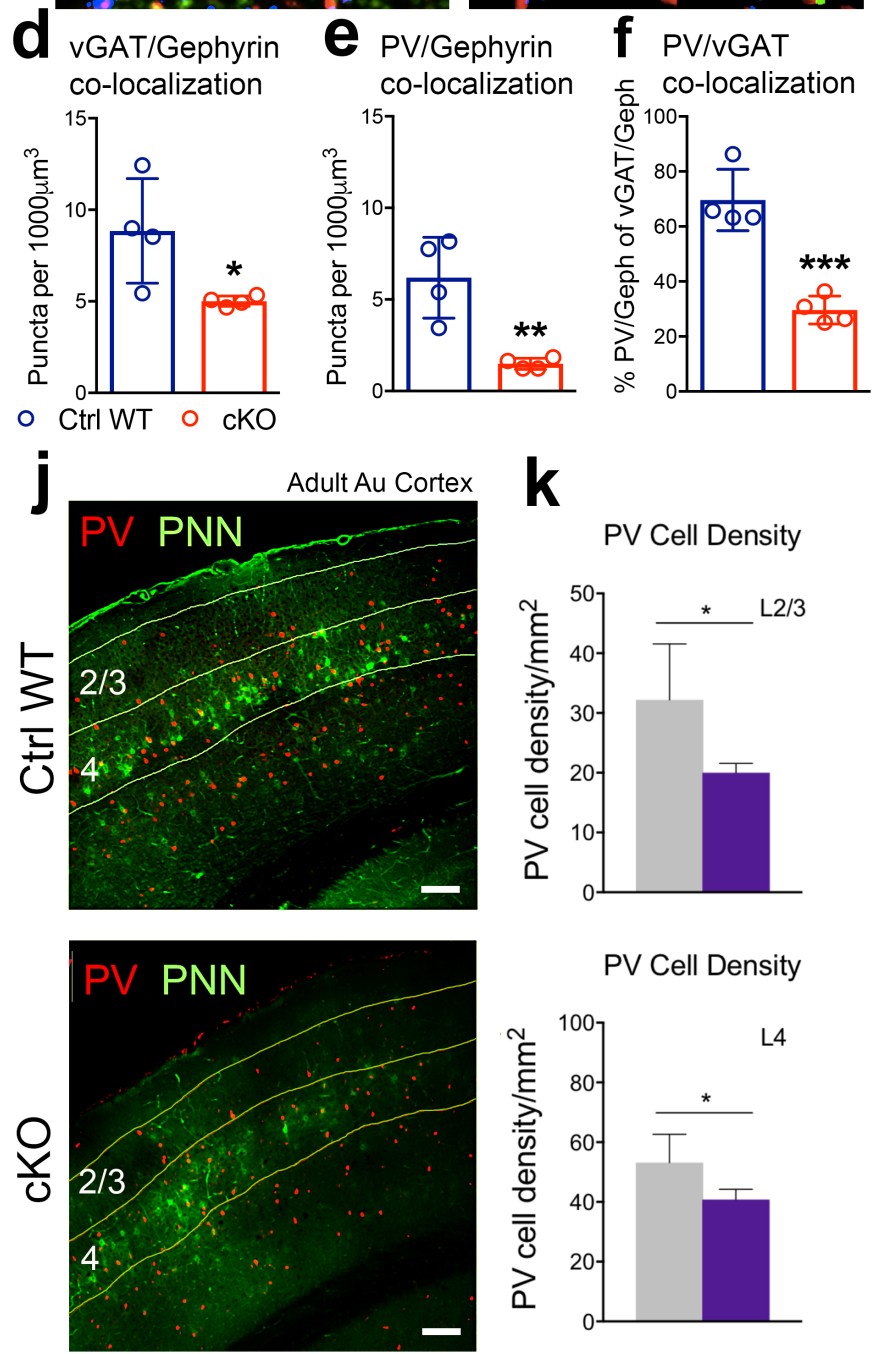

\section{$\mathbf{k}$}

PV Cell Density

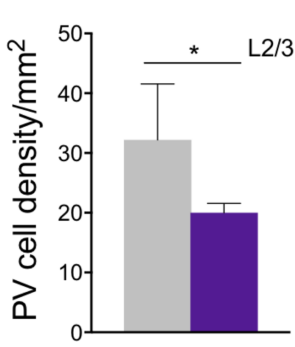

PV Cell Density

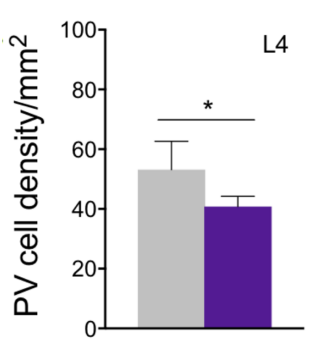

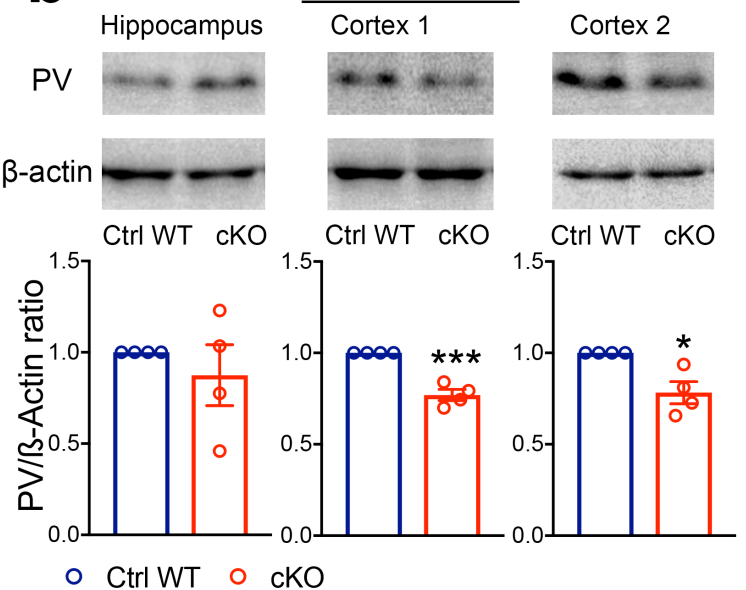

9 Excitatory synapses on PV
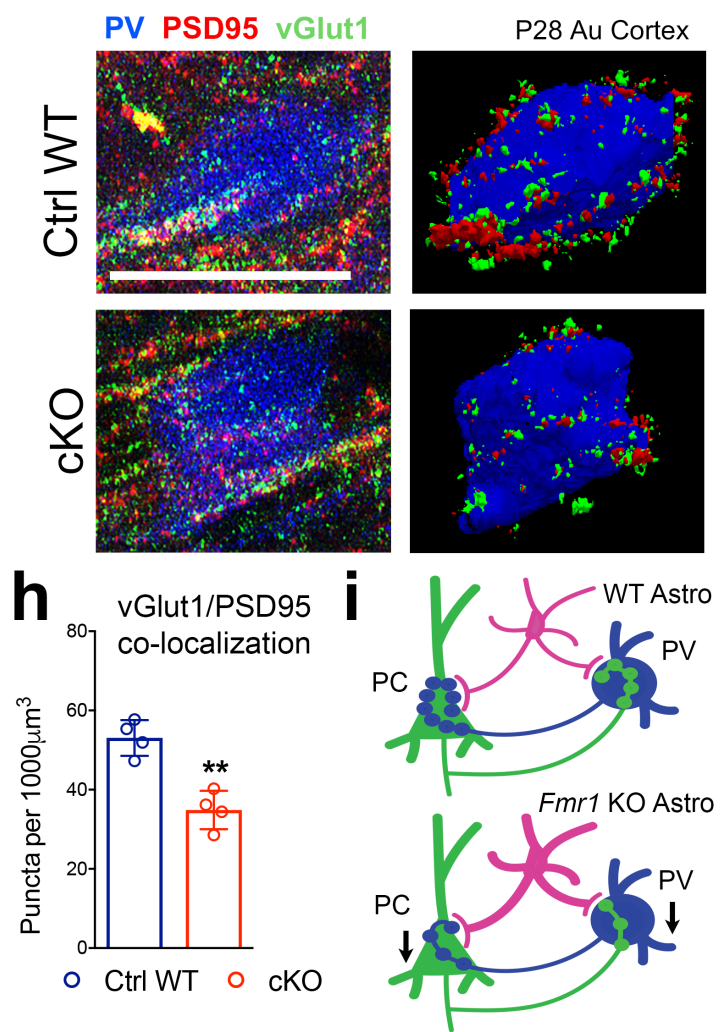

PNN Cell Density

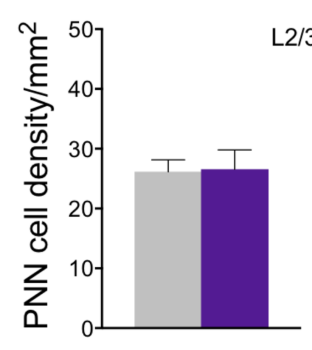

PNN Cell Density

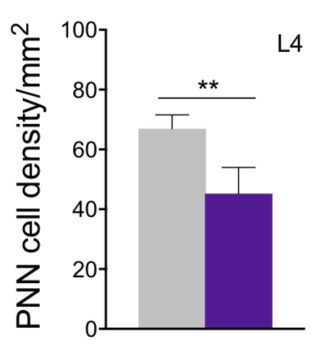

$\mathrm{CKO}$

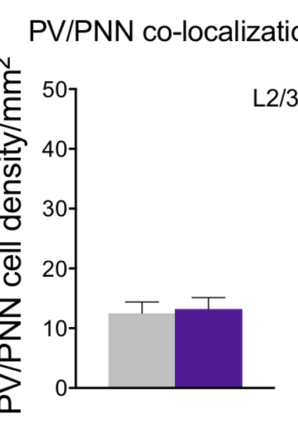

PV/PNN co-localization

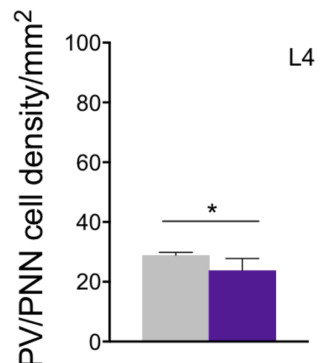


Figure 3

a $\quad$ Example Traces (Ctrl WT)
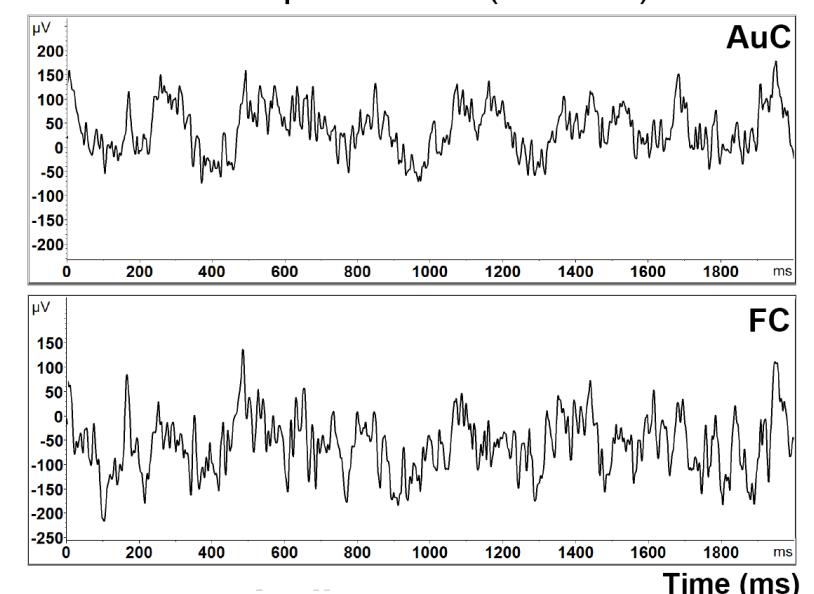

b

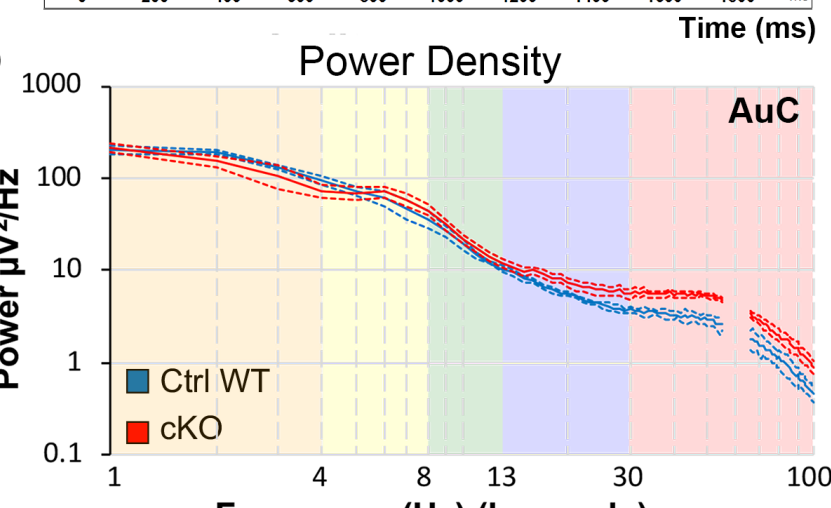

Frequency $(\mathrm{Hz})$ (log scale)
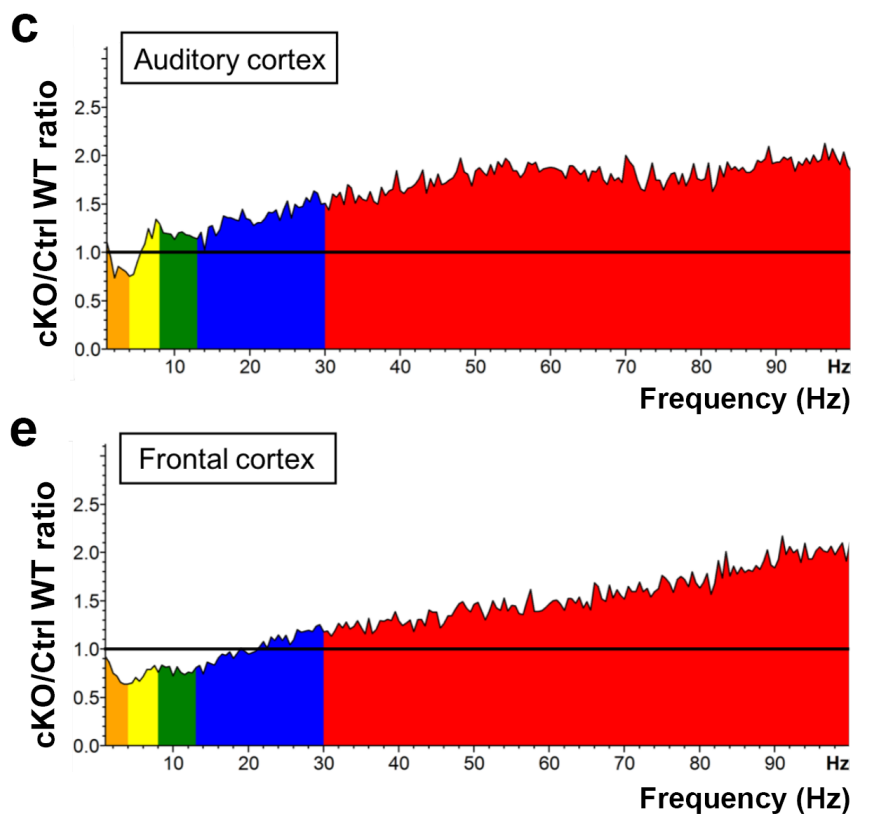

Delta

(1-4Hz)

$(4-8 \mathrm{~Hz})$

Alpha

$(8-13 \mathrm{~Hz})$

d $2.5 \mathrm{AuC}$

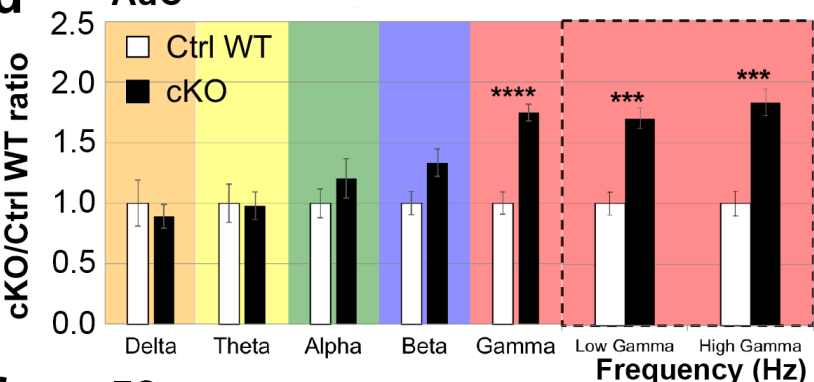

f $2.5 \mathrm{FC}$

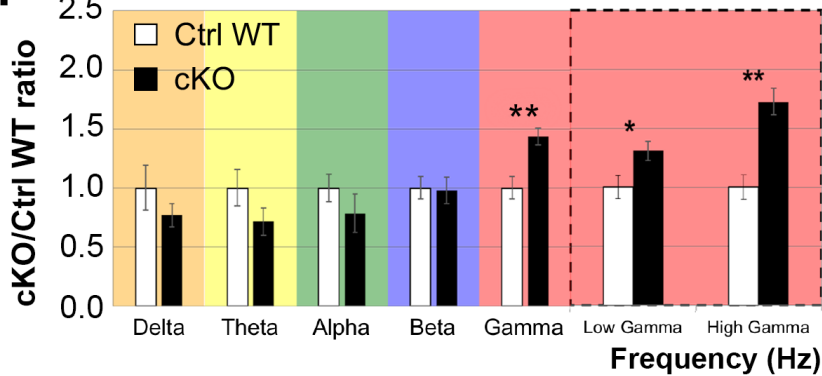

Gamma

Beta

$(13-30 \mathrm{~Hz})$

$(30-100 \mathrm{~Hz})$ g Power Coupling

AuC FC

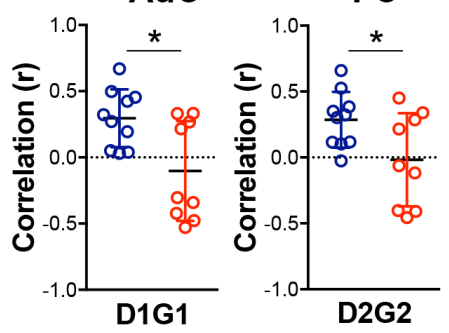

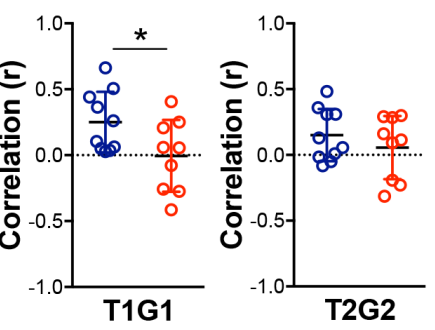

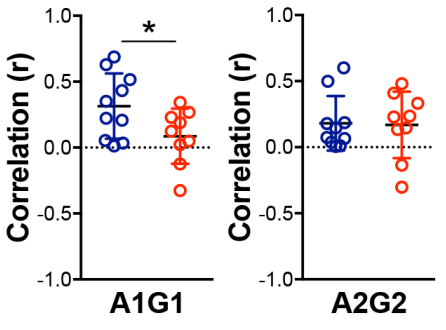

AuC - FC FC - AuC

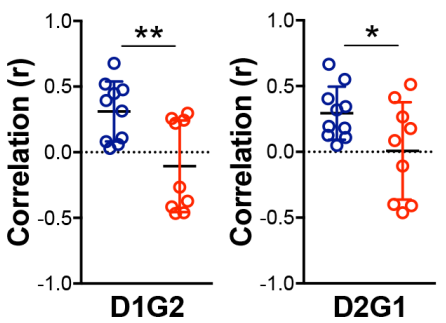

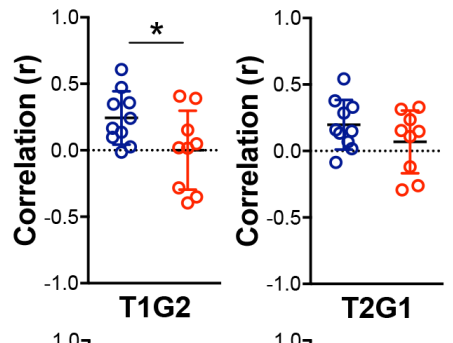

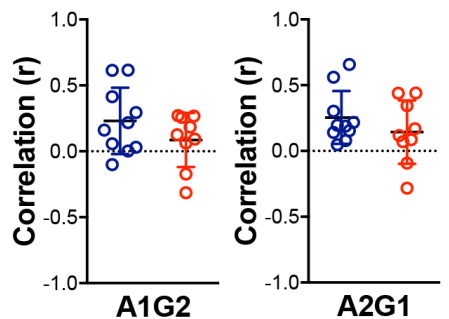


Figure 5

a Example Traces $(0.25 \mathrm{~Hz}$ single $)$

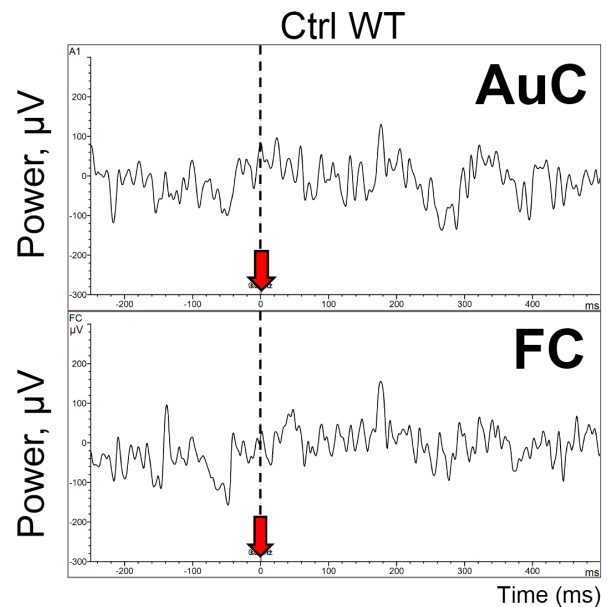

C Induced Power (0.25 Hz single)

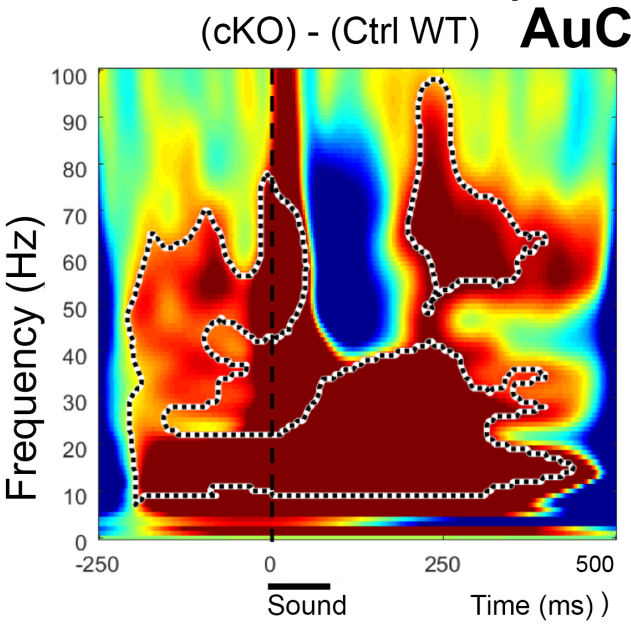

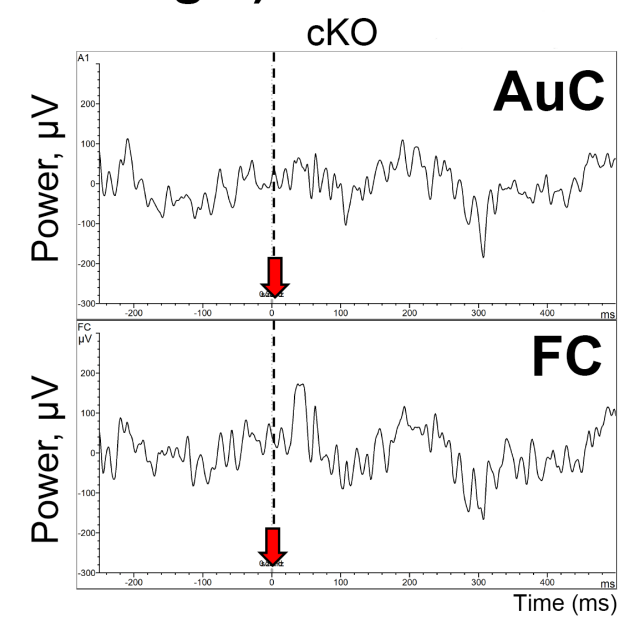

(cKO) - (Ctrl WT) FC

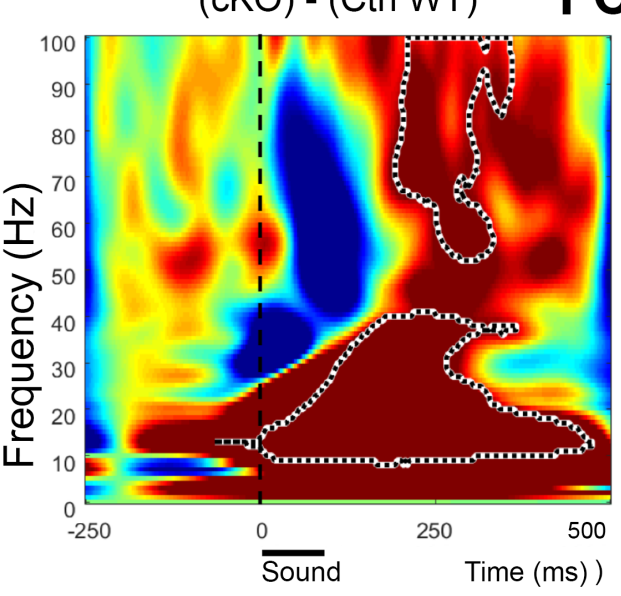

b Example Traces (4Hz train)

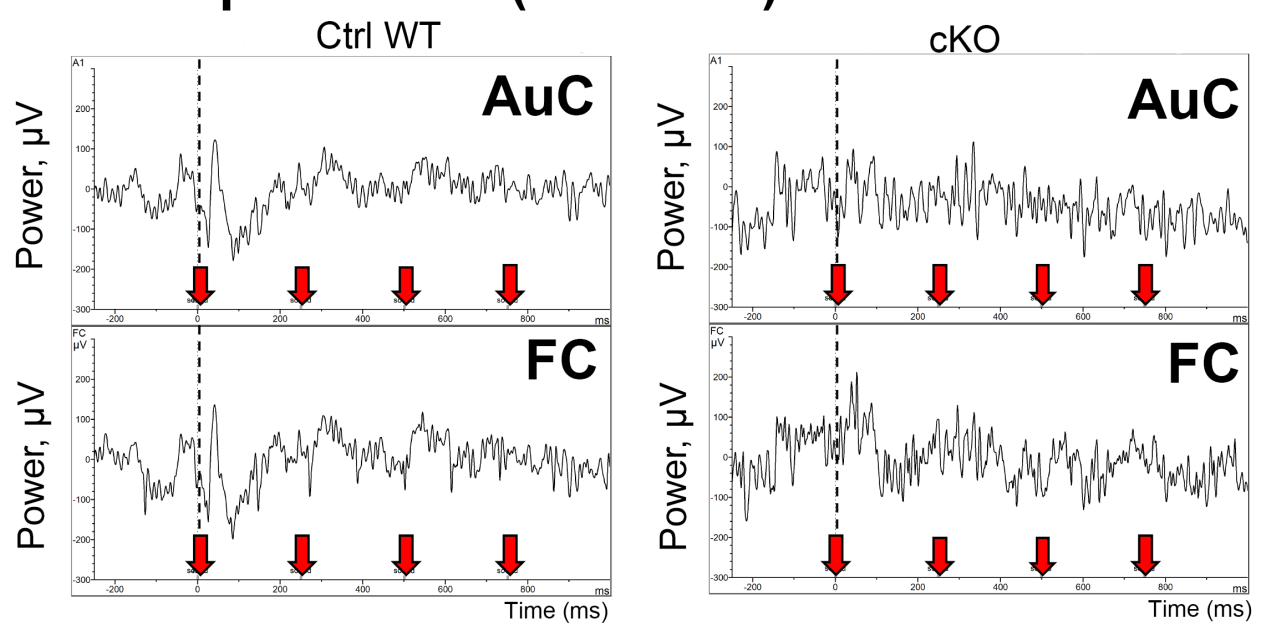

d Induced Power (4Hz train)

(cKO) - (Ctrl WT) AuC

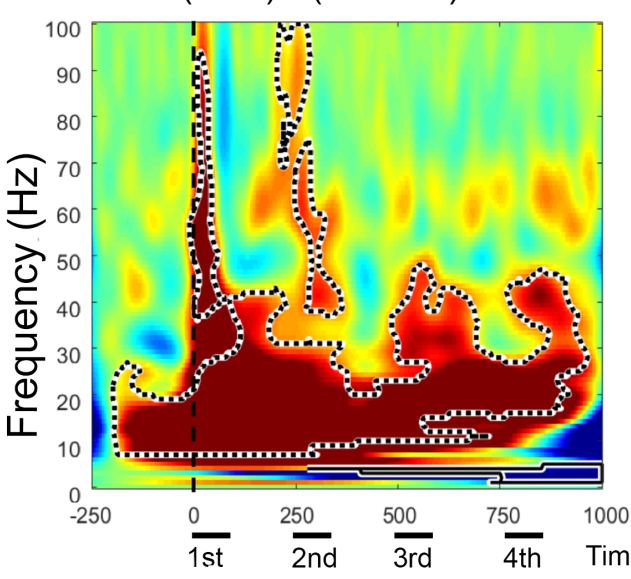

(cKO) - (Ctrl WT)

FC

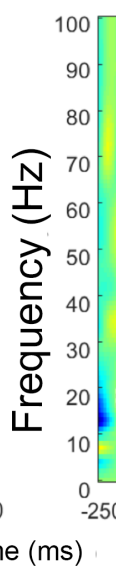




\section{Figure 6}

\section{a Heatmap of GABAergic proteins}

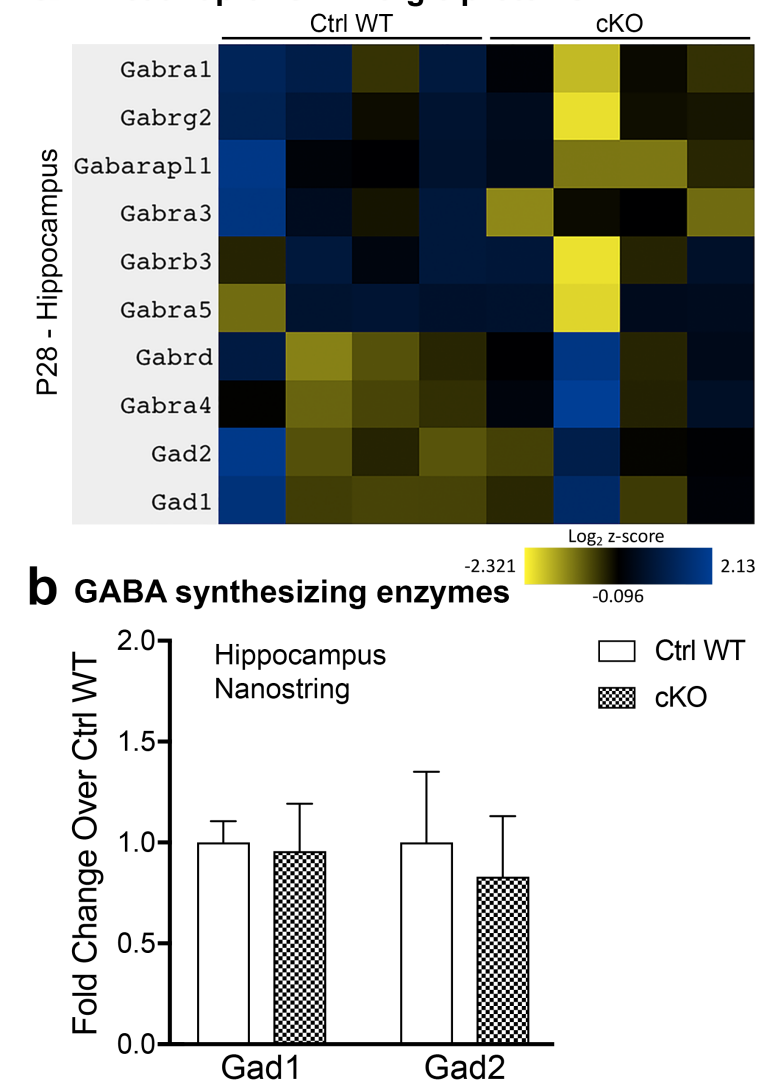

\section{Synaptic}

C.1. Gabra1
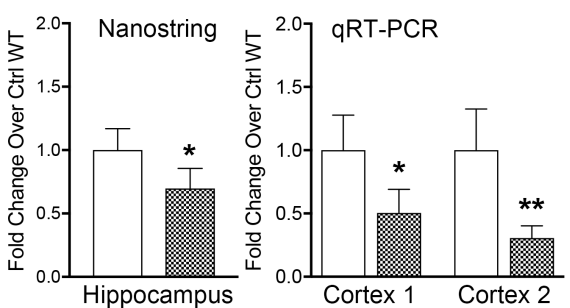

C.2. Gabra3
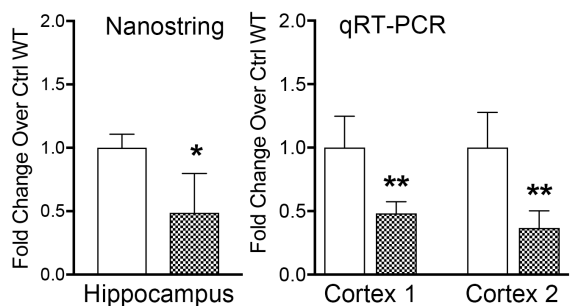

C.3. Gabrg2

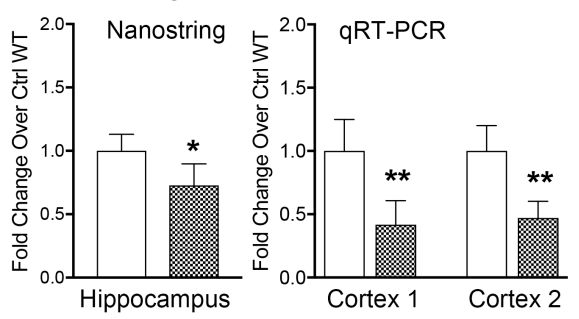

\section{d Extrasynaptic}

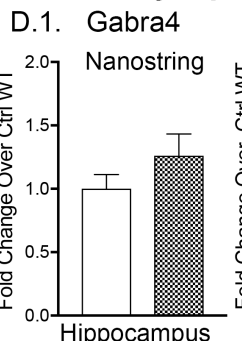

D.2. Gabra5
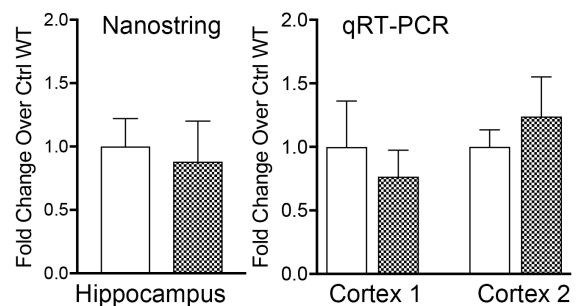

D.3. Gabrd
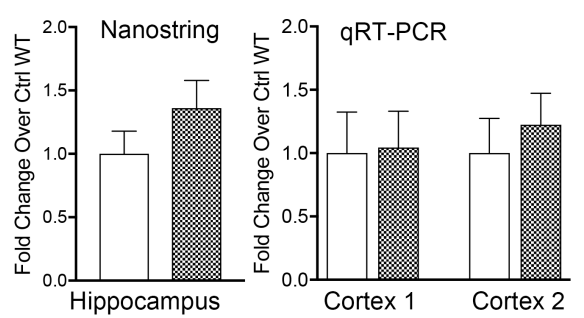

e Western Blot

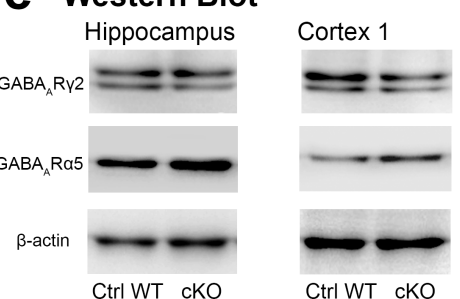

f GABA $_{\mathrm{A}}$ RY2 Protein Levels
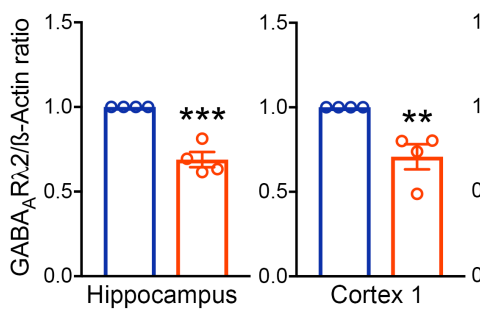

○ Ctrl W实富

0 Ctrl WT

15 $\bigcirc$ CKO

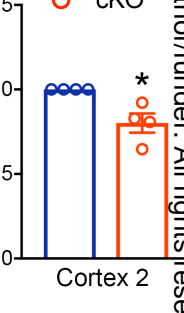

\section{GABA $_{A}$ Ra5 Protein Levels}

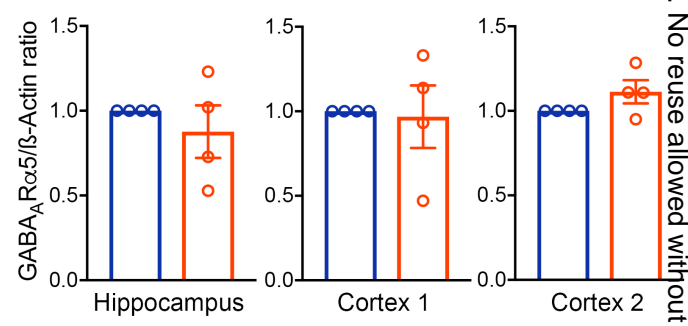




\section{Figure 7}

a GABA immunoreactivity
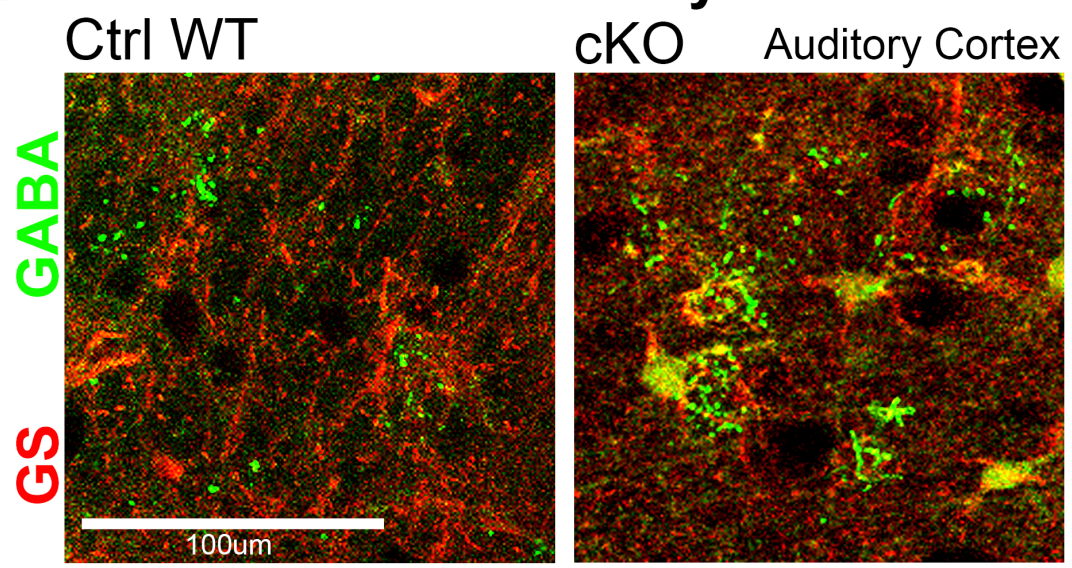

b GABA levels in GS-labeled astrocytes

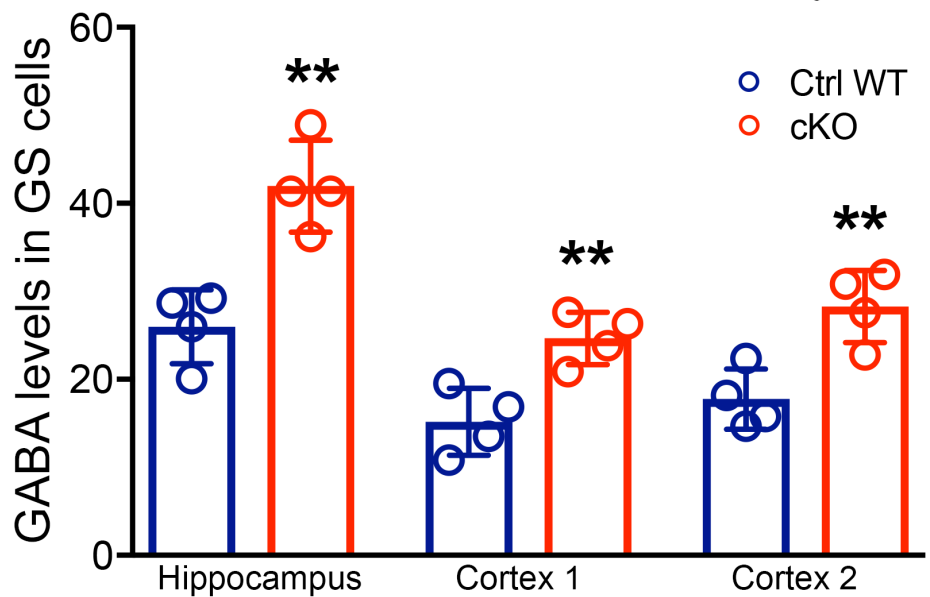

C Immunoblot: protein levels in astrocytes
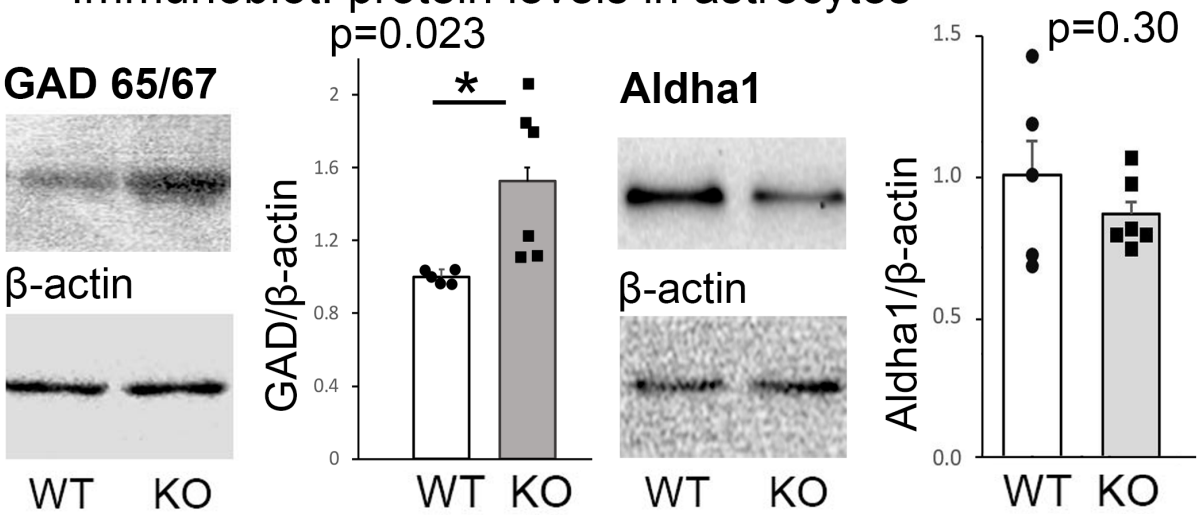

d GABA levels with
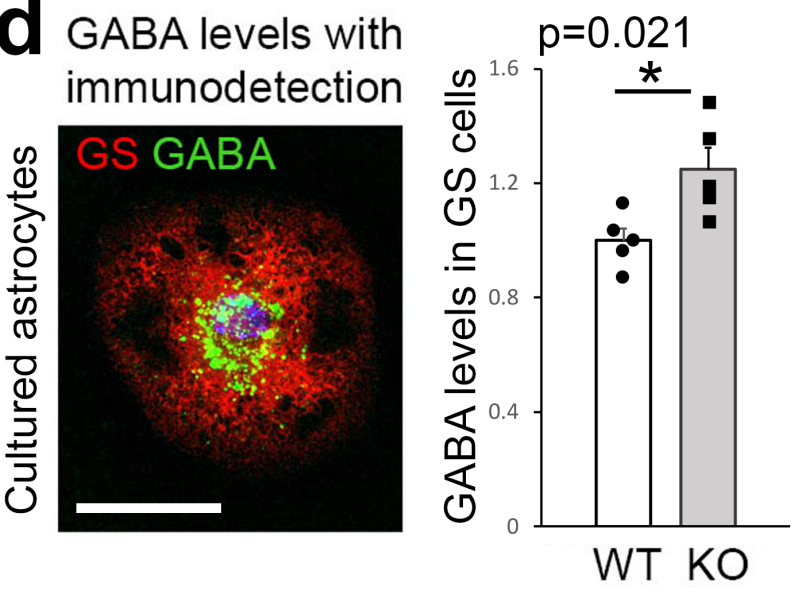

e GFAP

$\underline{\text { mRNA Levels }}$

f

Protein Levels

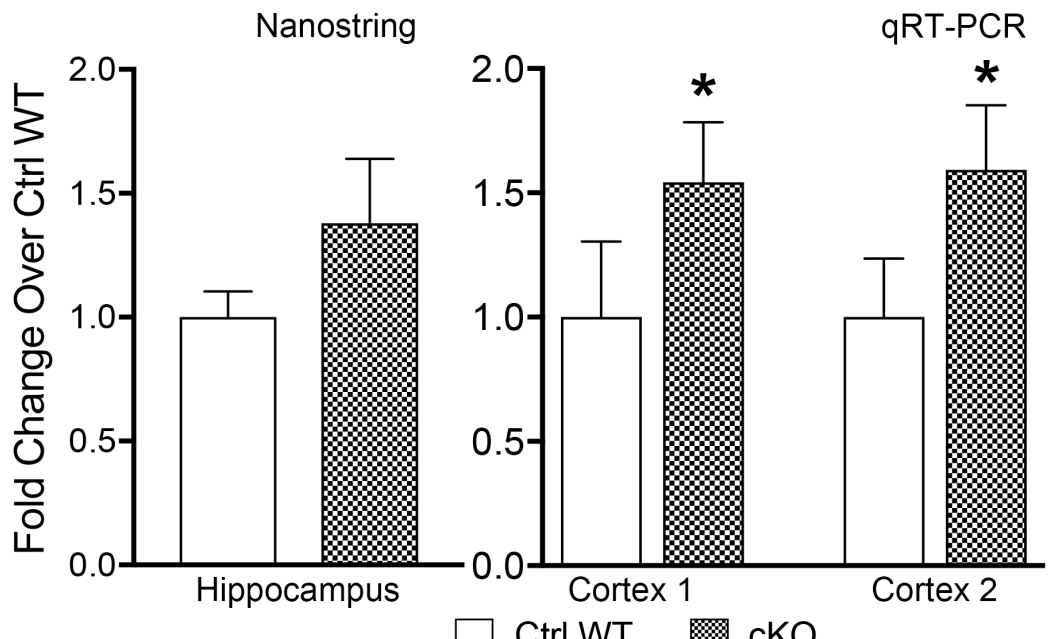

GFAP

$\beta$-actin

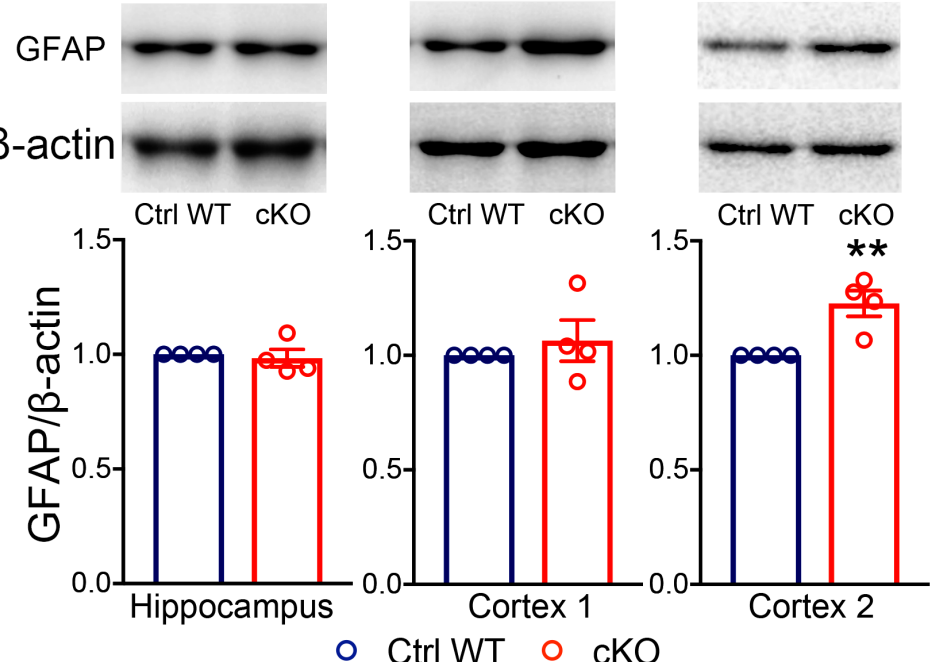




\section{Elevated Plus Maze Open Field}

a Locomotor Activity

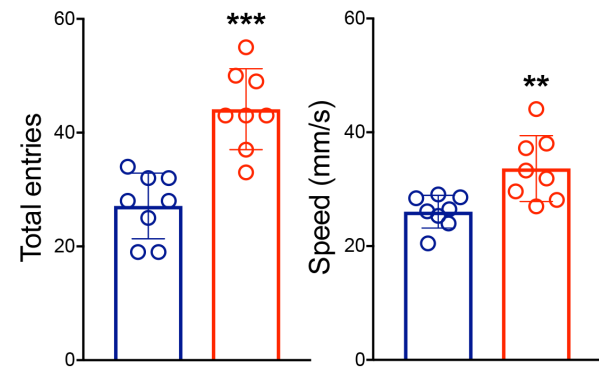

b Anxiety-like Behavior
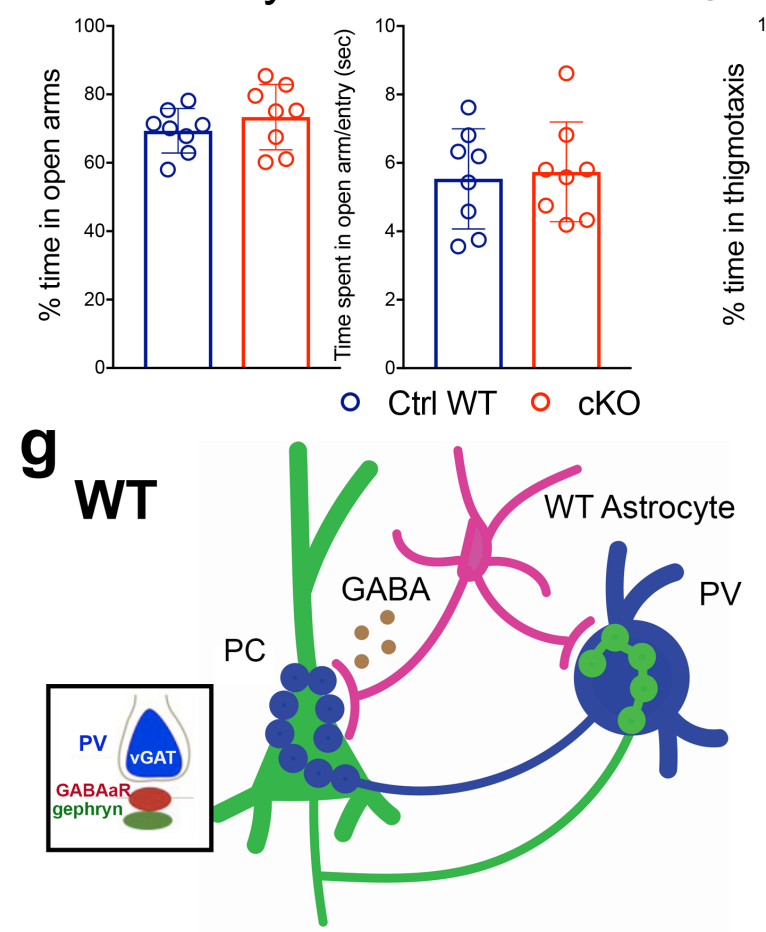

d Anxiety-like Behavior

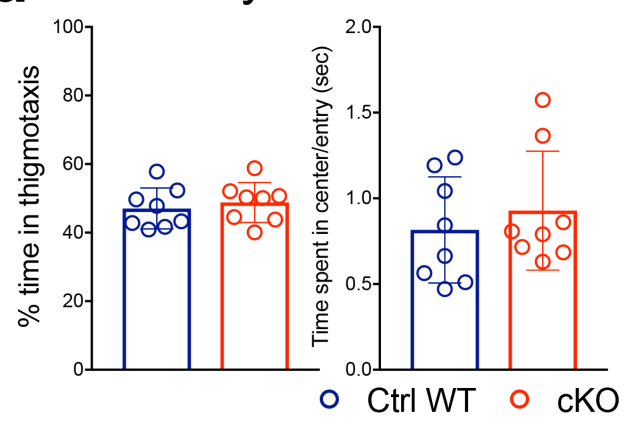

c Locomotor Activity

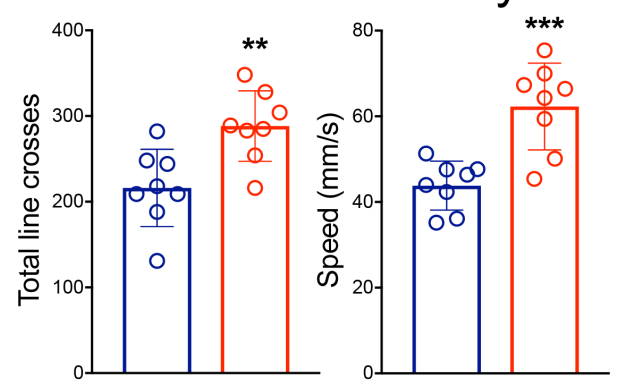

Social Novelty Test

e Sociability

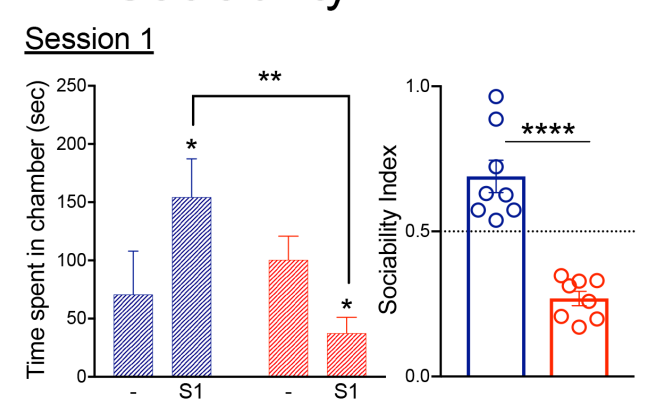

f Social Novelty

Session 2

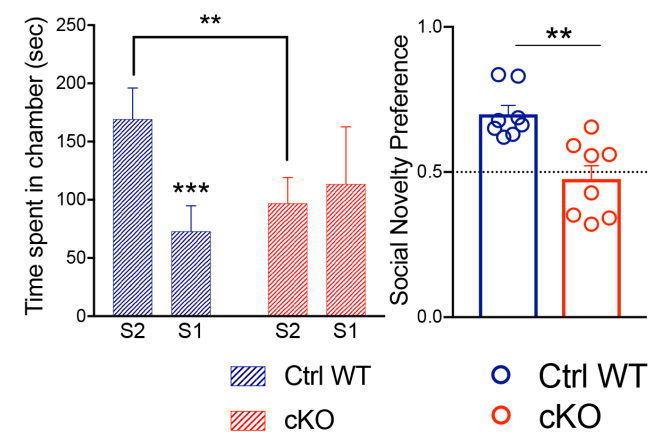

Gamma synchronization ERP power

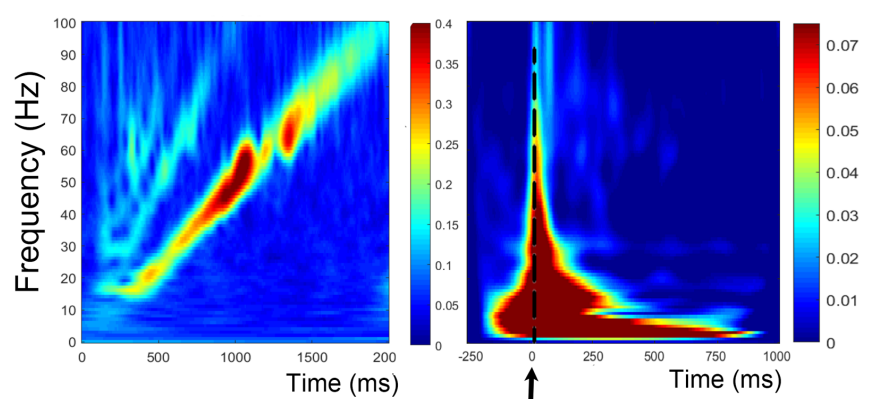

Normal behaviors
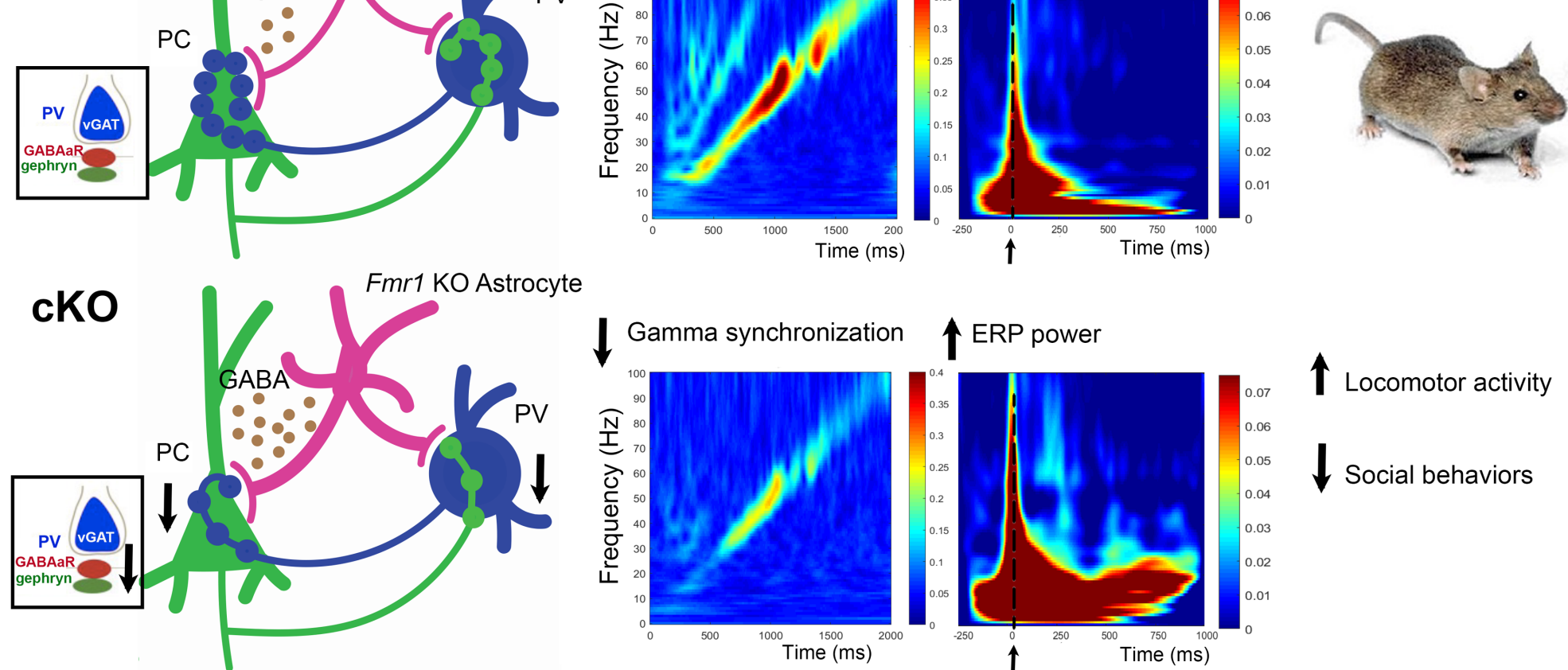

$\downarrow$ Gamma synchronization $\uparrow$ ERP power
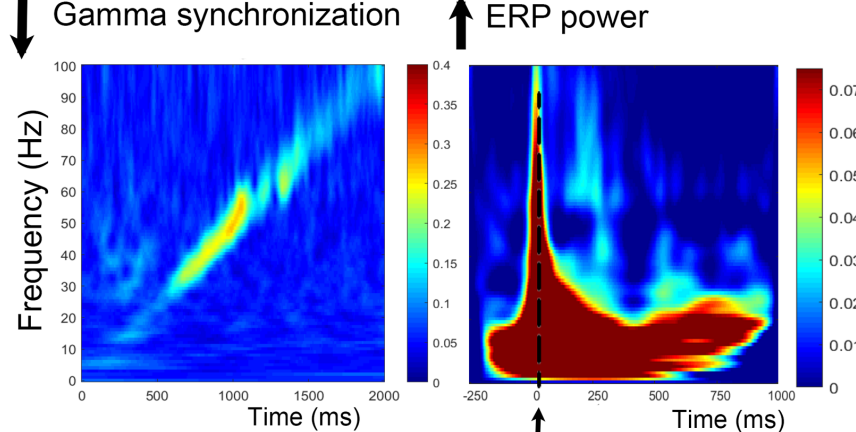

$\uparrow$ Locomotor activity

$\downarrow$ Social behaviors 